\title{
Activity-related variations of high-degree p-mode amplitude, width, and energy in solar active regions
}

\author{
R. A. Maurya ${ }^{1,2}$, A. Ambastha ${ }^{2}$, and J. Chae ${ }^{1}$ \\ 1 Department of Physics and Astronomy, Seoul National University, 151-747 Seoul, Republic of Korea \\ e-mail: ramajor@astro.snu.ac.kr; jcchae@snu.ac.kr \\ 2 Udaipur Solar Observatory, Physical Research Laboratory, 313001 Udaipur, India \\ e-mail: ambastha@prl.res.in
}

Received 6 February 2013 / Accepted 14 November 2013

\begin{abstract}
Context. Solar energetic transients such as flares and coronal mass ejections occur mostly within active regions (ARs) and release large amounts of energy, which is expected to excite acoustic waves by transferring the mechanical impulse of the thermal expansion of the flare on the photosphere. On the other hand, strong magnetic fields of AR sunspots absorb the power of the photospheric oscillation modes.

Aims. We study the properties of high-degree p-mode oscillations in flaring and dormant ARs and compare them with those in corresponding quiet regions (QRs) to find the association of the mode parameters with magnetic- and flare-related activities.

Methods. We computed the mode parameters using the ring-diagram technique. The magnetic-activity indices (MAIs) of ARs and QRs were determined from the line-of-sight magnetograms. The flare indices (FIs) of ARs were obtained from the GOES X-ray fluxes. Mode parameters were corrected for foreshortening, duty cycle, and MAI using multiple non-linear regression.

Results. Our analysis of several flaring and dormant ARs observed during the Carrington rotations 1980-2109 showed a strong association of the mode amplitude, width, and energy with magnetic and flare activities, although their changes are combined effects of foreshortening, duty cycle, magnetic-activity, flare-activity, and measurement uncertainties. We find that the largest reduction in mode amplitude and background power of an AR are caused by the angular distance of the AR from the solar disc centre. After correcting the mode parameters for foreshortening and duty cycle, we find that the mode amplitudes of flaring and dormant ARs are lower than in corresponding QRs reducing with increasing MAI, suggesting a stronger mode power suppression in ARs with larger magnetic fields. The mode widths in ARs are larger than in corresponding QRs and increase with MAI, indicating shorter lifetimes of modes in ARs than in QRs. The variations in mode amplitude and width with MAI are not same in different frequency bands. The largest amplification (reduction) in mode amplitude (mode width) of dormant ARs is found in the five-minute frequency band. The average mode energy of both the flaring and dormant ARs is smaller than in their corresponding QRs, reducing with increasing MAI. But the average mode energy reduction rate in flaring ARs is smaller than in dormant ARs. Moreover, the increase in mode width rate in dormant (flaring) ARs is followed by a decrease (increase) in the amplitude variation rate. Furthermore, including the mode corrections for MAI shows that mode amplitude and mode energy of flaring ARs escalate with FI, while the mode width shows an opposite trend, suggesting excitations of modes and growth in their lifetimes by flares. The increase (decrease) in mode amplitude (width) is larger in the five-minute and higher-frequency bands. The enhancement in width variation rate is followed by a rapid decline in the amplitude variation rate.
\end{abstract}

Key words. Sun: helioseismology - Sun: magnetic field - Sun: activity - Sun: flares

\section{Introduction}

Photospheric five-minute oscillations, probably first observed by Leighton et al. (1962), are caused by trapped acoustic waves (p-modes) inside the solar interior (Ulrich 1970; Leibacher \& Stein 1971) and are well known and have been studied extensively. It is believed that the energy of p-modes is contributed by convective or radiative fluxes. A precise determination of the p-modes properties provides a powerful tool to probe the solar interior. High-degree $(\ell>200)$ acoustic oscillations are vertically trapped in a spherical shell with the photosphere as the upper boundary and the lower boundary depending on the horizontal wavenumber, $k_{\mathrm{h}}^{2}=\frac{\ell(\ell+1)}{r^{2}}$, and the frequency $(\omega)$,

$\frac{\ell(\ell+1)}{r_{\mathrm{t}}^{2}}=\frac{\omega^{2}}{c_{s}^{2}\left(r_{\mathrm{t}}\right)}$,

where $r_{\mathrm{t}}$ is depth of the lower turning point. Lifetimes of highdegree modes are much shorter than the sound travel time around the Sun, therefore local effects are more important for these modes than for the low-degree modes, which have longer horizontal wavelengths and longer lifetimes. It is likely that highdegree acoustic waves are not global modes, that is, they do not remain coherent while travelling over the circumference to interfere with themselves. Therefore, they can locally be considered as horizontally travelling, vertically trapped waves. These are observed as photospheric motions inferred from the Doppler shifts of photospheric spectral lines. The analysis of local modes provides a diagnostic tool to study the structural and dynamic properties of the solar interior beneath ARs as well as the changes in excitation and damping of modes that are most affected by the surface magnetic fields.

Previous studies showed that characteristics of the highdegree modes are modified in active regions (ARs) with complex and strong magnetic fields associated with sunspots, which are different from magnetically quiet regions (QRs). The significant reduction of the p-mode energy in ARs has been 
attributed to absorption by sunspots (Braun et al. 1987; Braun \& Duvall 1990; Bogdan et al. 1993; Zhang 1997; Hindman \& Brown 1998; Haber et al. 1999; Mathew 2008; Gosain et al. 2011). Previous studies showed that the mode power absorption changes with harmonic degree and radial order. The absorption increases with increasing horizontal wavenumber $k_{\mathrm{h}}$ over the range $0.0-0.8 \mathrm{Mm}^{-1}$ and decreases for higher $k_{\mathrm{h}}$ in the range $0.8-1.5 \mathrm{Mm}^{-1}$. The absorption along each individual p-mode ridge tends to peak at an intermediate value of the degree 200-400. Hindman \& Brown (1998) found that the amplitudes of oscillations with frequencies lower than $5.2 \mathrm{mHz}$ decrease with field strength for both velocity and continuum intensity measurements. Furthermore, they reported that oscillations with frequencies between 5.2 and $7.0 \mathrm{mHz}$ within ARs suppressed continuum intensity amplitudes, but enhanced velocity amplitudes.

The differences in mode frequency and width in ARs and QRs have been studied earlier. Rajaguru et al. (2001) found that the frequencies of solar oscillations are significantly higher in ARs than in QRs. Width and asymmetry of the peaks in power spectra are also larger in ARs, while the mode amplitudes are smaller. Furthermore, Howe et al. (2004) showed that the difference in mode characteristics are correlated with the differences in the average surface magnetic fields between corresponding regions. They reported a strong dependence of amplitude and lifetime of $\mathrm{p}$-modes on the local magnetic flux, and found that these parameters decreased in the five-minute band, while a reverse trend was found at high frequencies. Rabello-Soares et al. (2008) confirmed previous results and reported that mode amplitude and width variations are nearly linear.

Changes in the mode parameters with solar activity cycle have also been studied by many researchers. Tripathy et al. (2010) found that changes in mode frequency during the activityminimum period are significantly stronger than during the solar maximum. Komm et al. (2000a) reported a 23\% increase in mode widths (i.e., decrease in mode lifetimes) with increasing solar activity. They found that the variations are frequency dependent; they are strongest near $3.1 \mathrm{mHz}$, and are independent of harmonic degree $\ell$. Changes in mode amplitudes, power, and energy-supply rate were analysed from solar minimum to maximum. It was found that mode amplitudes and solar activity level are anti-correlated for intermediate and high-degree modes, and strongly depend on local magnetic fields. Chaplin et al. (2000) suggested that the changes in mode parameters arise from changes in damping instead of excitation. Burtseva et al. (2009a) found that the mode lifetime in ARs and QRs decreases with magnetic-activity, but the decrease is slower in QRs. Moreover, p-mode amplitudes at solar minimum are higher than at solar maximum (Burtseva et al. 2009b).

Energetic transients, viz., flares, and coronal mass ejections (CMEs), are believed to be caused by reconnection of magnetic fieldlines in the solar atmosphere. Particles are energized at the primary energy-release site in the corona and then guided along the magnetic fieldlines downwards to the denser atmosphere. They are expected to affect and excite the high-degree p-modes by transferring mechanical impulse of thermal expansion on the photospheric layer (Wolff 1972). The observational evidence of flare-induced horizontally travelling waves on the solar photosphere has been reported previously (Kosovichev \& Zharkova 1998; Donea et al. 1999; Donea \& Lindsey 2005; Kosovichev 2006, 2011). The detection of seismic waves provides us with a unique opportunity to study the excitation of solar oscillations, and raise new questions about the underlying physical processes as well as the properties of the excited waves and source producing them. But the observations of seismic waves are relatively rare, possibly because of the difficulties of detecting the photospheric ripples. Such travelling waves appear to be associated mostly with energetic flares.

Flare-related variations in high $\ell$ p-mode parameters have been studied previously (Ambastha et al. 2003; Maurya et al. 2009; Maurya 2010; Maurya \& Ambastha 2011). Ambastha et al. (2003) have found that power in high-degree p-modes is stronger amplified during periods of high flare-activity in some ARs than in the non-flaring ARs of similar magnetic fields. Maurya et al. (2009) found strong evidence of a substantial increase in mode amplitude during an extremely energetic flare. On the other hand, at a global scale of the whole solar disc, Ambastha \& Antia (2006) found a poor correlation between the running mean of FI and low- $\ell$ mode power. They reported similar changes for the CME index. Karoff \& Kjeldsen (2008) found that energy in the flare can drive global oscillations in the Sun in the same way as the Earth is set ringing after a major earthquake. They reported a stronger correlation between flares and oscillation energy at high frequencies than for the ordinary p-modes. Flare-induced excitations at higher frequencies were also reported by Kumar et al. (2010, 2011). A more recent study by Richardson et al. (2012), however, did not find evidence of flare-driven high-frequency global modes. These high-frequency modes, unlike the p-modes, are not expected to be trapped in the solar interior.

Solar ARs, where the flares typically occur, are locations of strong magnetic fields associated with the sunspots. Thus, there is a competition between the absorption of p-mode energy by sunspots and excitation/amplification by the energetic transients. The net increase or decrease of the mode energy would, therefore, depend on their relative dominance. Characteristics of the photospheric oscillations in ARs are essentially described by the modes of shorter wavelength, which are trapped just below the photosphere. These modes can be studied using the so-called ring-diagram analysis (Hill 1988), which is a wellestablished technique in local helioseismology. It is a generalization of the global-mode analysis for small patches of the solar surface, where the geometry is approximately plane parallel, and waves can be treated as plane waves. The modes measured using this technique are typically $\ell>150$, which have a lower turning depth above $r / R_{\odot} \approx 0.95$. For more details about this technique, we refer to the review by Antia \& Basu (2007).

The ring-diagram technique uses several hours-long timeseries of Doppler observation which may dilute the shorterduration flare-induced effects owing to the averaging involved in space and time. Nevertheless, we found strong evidence of flare-related amplification in p-mode amplitude during the longduration, high magnitude X17.2/4B flare of 28 October 2003 (Maurya et al. 2009). This study illustrated the possibility of detecting changes in p-mode parameters for long-duration energetic flares with a ring-diagram analysis. The observed amplification in p-mode amplitude implies that during an energetic flare, the energy of p-modes must be weighted by the energy of excited modes. To further establish these results, we investigate several ARs, both flare producing and dormant along with QRs. We undertake a set of 53 flaring ARs of varying magnetic complexity to examine the relationship of mode amplitude, width, and energy with magnetic fields and flare energies. However, the ring-diagram analysis can also be used to study the frequency shift, sub-photospheric flow, sound speed, etc.

The paper is organized as follows: Sect. 2 describes the selected sample of flaring and dormant ARs and the observational data used for our study. The methods of data analysis and the 
R. A. Maurya et al.: Variations of p-mode amplitude, width, and energy in active regions

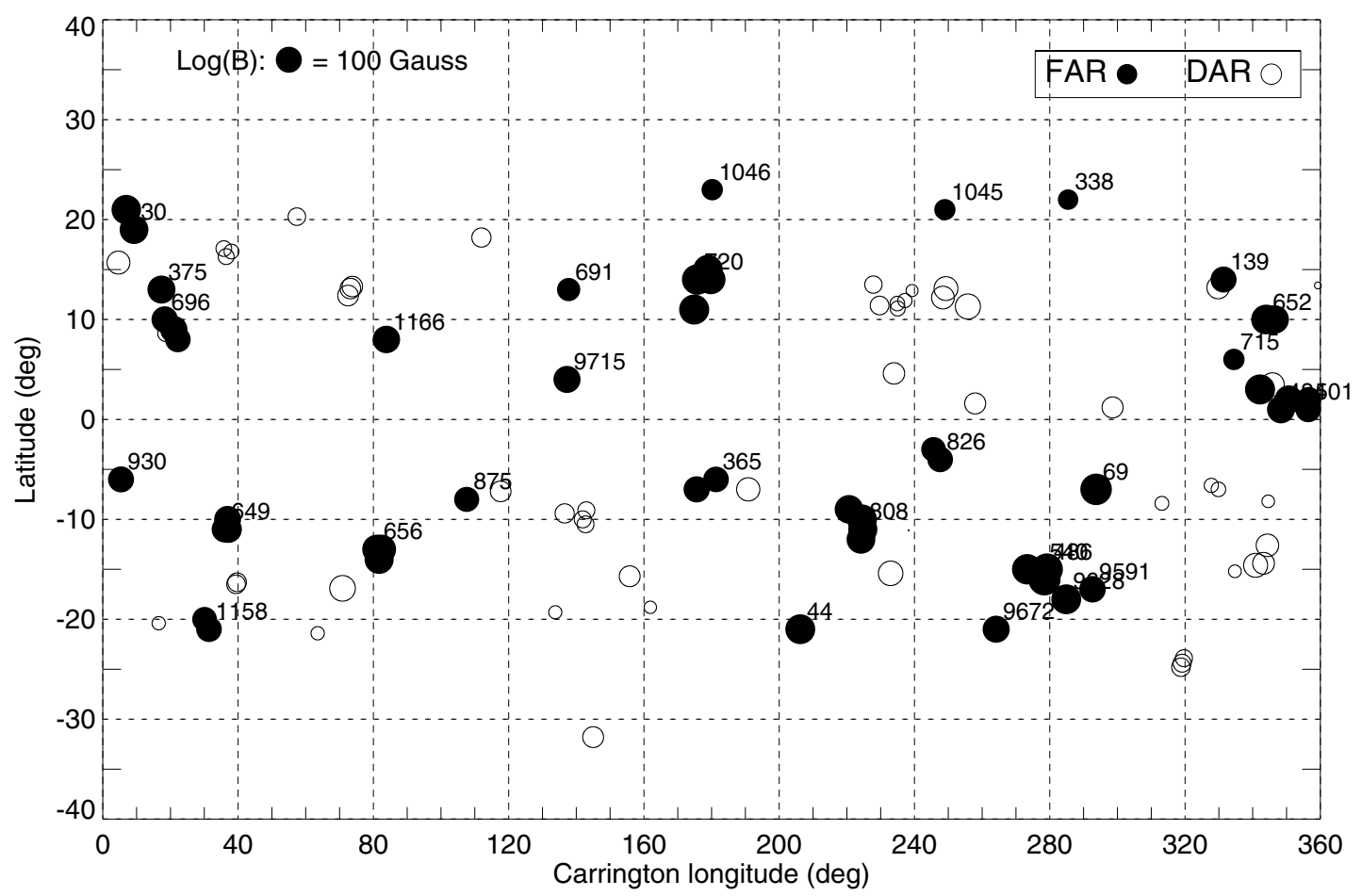

Fig. 1. Distribution of flaring (filled circle) and dormant (unfilled circle) ARs used in this study as shown in a Carrington map. Circle sizes correspond to the magnetic-activity index (B; for detail see Sect. 3.3) of ARs in Gauss.

results are given in Sect. 3. We briefly discuss how the mode parameters are affected by foreshortening, duty cycles, and magnetic activities, and present an empirical method to correct them. We assumed that these effects are the only systematic variations and that measurement uncertainties are essentially random and are compensated for by averaging over many data sets. In Sect. 4 we present the results of the analysis and discuss them. We study variations in mode parameters with magnetic and flare-activity indices of ARs. We present average properties of the mode parameters and not their temporal variations. Section 5 gives the summary and conclusions.

\section{Active regions and observational data}

\subsection{Flaring and dormant active regions}

We selected our sample of energetic events occurring in different ARs using the archived information on ARs and solar activity from the web-pages of the Solar Monitor ${ }^{1}$. We first identified flares of X-ray classes >M5.0 during the period of Carrington rotations 1980-2109 of solar cycles 23 and 24. We short-listed the ARs lying within the angular distance of $40^{\circ}$ from the solar disc centre and selected 53 events that were well covered by the Global Oscillation Network Group (GONG) project. Out of 53 events, 48 events correspond to solar cycle 23 and 5 to the current solar cycle 24 . We also selected suitable QRs corresponding to every flaring AR located at the same latitude but at a different Carrington longitude.

To study the flare-induced excitation in flaring ARs as compared with non-flaring ARs, we also selected dormant ARs and associated QRs as for the flaring ARs. Thus, for every flare event, we selected four regions: flaring and dormant ARs and their associated QRs for the same time period. The locations and distribution of flare events and corresponding ARs over the solar

\footnotetext{
1 http://www. solarmonitor.org
}

disc are illustrated in Fig. 1. This shows that most of the events selected in our sample occurred within the latitude zone of $\pm 20^{\circ}$. For brevity, throughout we used the notation $B$ for the magneticactivity index (MAI) of an AR, measured in Gauss, which is not to be confused with the magnetic-field strength.

\subsection{Observational data}

The observational data used in this study consist of merged Dopplergrams at one-minute cadence, obtained by GONG (Harvey et al. 1988), 96-min averaged magnetograms from the Michelson Doppler Imager (MDI, Scherrer et al. 1995) on board the Solar and Heliospheric Observatory (SOHO), and the X-ray fluxes from the GOES data archive. The pixel resolutions for GONG Dopplergrams and MDI magnetograms are 2.5" and $2.0^{\prime \prime}$, respectively. The $96-\mathrm{min}$ averaged magnetograms provided by MDI data archive are averages of calibrated one-minute magnetograms (with a noise level of $\sim 30 \mathrm{G}$ ) and five-minute magnetograms (with noise level of $\sim 15 \mathrm{G}$ ).

\section{Analysis techniques}

\subsection{Ring diagrams and $p$-mode parameters}

To estimate the p-mode parameters corresponding to a selected area over the Sun, the region of interest is tracked over time. This spatio-temporal area is defined by an array (or data cube) of dimension $N_{x} \times N_{y} \times N_{t}$. Here, first two dimension $\left(N_{x}, N_{y}\right)$ correspond to the spatial size of the AR along $x$ - and $y$-axes, representing zonal and meridional directions, and the third $\left(N_{t}\right)$ to the time $t$ in minutes. The data cubes employed for the ring diagram analysis have typically duration of $1664 \mathrm{~min}$ and cover area of $16^{\circ} \times 16^{\circ}$ centred around the location of interest. This choice of area is a compromise between the spatial resolution on the Sun, the range of depth and the resolution in spatial wavenumber 
of the power spectra. A larger size allows accessing the deeper sub-photospheric layers, but only with a coarser spatial resolution. On the other hand, a smaller size not only limits access to the deeper layers, but also renders the fitting of rings more difficult.

The spatial coordinates of pixels in tracked images are not always integer. To apply the three-dimensional Fourier transform on tracked data cube, we interpolated the coordinates of tracked images to integer values, for which we use the sinc interpolation method. Three-dimensional Fourier transformation of data cube truncates the rings near the edges due to the aliasing of higher frequencies toward lower side. To avoid the truncation effects, we apodized the data cube in both the spatial and temporal dimensions. The spatial apodization was obtained by a $2 \mathrm{D}$-cosine bell method, which reduces the $16^{\circ} \times 16^{\circ}$ area to a circular patch with a radius of $15^{\circ}$ (Corbard et al. 2003).

The observed photospheric velocity signal $v(x, y, t)$ in the data cube is a function of position $(x, y)$ and time $(t)$. Let the velocity signal in frequency domain be $f\left(k_{x}, k_{y}, \omega\right)$, where, $k_{x}$ and $k_{y}$ are spatial frequencies in $x$ - and $y$-directions, respectively, and $\omega$ is the angular frequency of oscillations. Then the data cube $v(x, y, t)$ can be written as

$v(x, y, t)=\iiint f\left(k_{x}, k_{y}, \omega\right) \mathrm{e}^{\mathrm{i}\left(k_{x} x+k_{y} y+\omega t\right)} \mathrm{d} k_{x} \mathrm{~d} k_{y} \mathrm{~d} \omega$.

The amplitude $f\left(k_{x}, k_{y}, \omega\right)$ of p-mode oscillations is calculated using three-dimensional Fourier transformation of Eq. (2). The power spectrum is given by

$P\left(k_{x}, k_{y}, \omega\right)=\left|f\left(k_{x}, k_{y}, \omega\right)\right|^{2}$.

The $16^{\circ}$ patch from GONG consists of 128 pixels, giving a spatial resolution $\Delta x=1.5184 \mathrm{Mm}$, that is, the $k$-number resolution, $\Delta k=3.2328 \times 10^{-2} \mathrm{Mm}^{-1}$, and Nyquist value and resolution for the harmonic degree $(\ell)$, approximately 1440 and 22.5 , respectively. The corresponding range in $k_{x}-k_{y}$ space is $(-2.069$, $2.069) \mathrm{Mm}^{-1}$. The temporal cadence and duration of the datasets give a Nyquist frequency of $8333 \mu \mathrm{Hz}$ and a frequency resolution of $10 \mu \mathrm{Hz}$, respectively.

To determine various p-mode parameters, the threedimensional power spectrum is fitted using the Lorentzian function (Haber et al. 2002; Hill et al. 2003) given by

$P\left(k_{x}, k_{y}, \omega\right)=\frac{A^{\prime}}{\left(\omega-\omega_{0}+k_{x} U_{x}+k_{y} U_{y}\right)^{2}+\Gamma^{2}}+\frac{b_{0}}{k^{3}}$,

where, $\left.k=\sqrt{(} k_{x}^{2}+k_{y}^{2}\right)$ is the total horizontal wavenumber. It can be identified with the degree $(\ell)$ of a spherical harmonic mode of global oscillations by $\ell(\ell+1)=k^{2} R_{\odot}^{2}$. The six fitting parameters, viz., zonal velocity $\left(U_{\mathrm{x}}\right)$, meridional velocity $\left(U_{\mathrm{y}}\right)$, background power $\left(b_{0}\right)$, mode central frequency $\left(\omega_{0}\right)$, mode width $(\Gamma)$, and the parameter $A^{\prime}$ are determined by the maximum-likelihood approach (Anderson et al. 1990). Note that $A^{\prime}$ is not the amplitude (or mode height); the amplitude $(A)$ can be determined from the expression, $A^{\prime}=A \times \Gamma^{2}$. For convenience we use the term "mode" to refer to these parameters, although they are not discrete modes in the same sense as those observed at lower degrees. We are here mainly interested in the flare- and magneticactivity associated changes in mode parameters: $A, b_{0}, \Gamma$, and mode energy (see Sect. 3.2).

A sample of p-mode parameters computed using the above method is shown in Fig. 2 for AR NOAA 10649 obtained for the data cube on 17 July 2004. Panel a shows the well-known $\ell-v$ diagram, which represents the dispersion relation of the acoustic waves trapped in the sub-photospheric depths of the AR.
Different ridges correspond to modes of different radial orders, $n=0, \ldots, 5$. Modes with higher harmonic degree $\ell$ correspond to shorter wavelengths, which are trapped in shallower depths (see Eq. (1)). These modes are more likely to be modified by the near surface activities, such as flares and sunspot-magnetic fields. Other panels show the natural logarithm values of the mode parameters, $A^{\prime}$, mode width $(\Gamma)$, and background power $\left(b_{0}\right)$ as a function of frequency.

\subsection{The $p$-mode energy}

Photospheric p-mode parameters provide a diagnostic tool to study sub-photospheric structures and dynamics of ARs. For instance, mode width and amplitude provide clues to the excitation and damping mechanisms of solar oscillations. The line width $(\Gamma)$ is directly related to the lifetime, $(2 \pi \Gamma)^{-1}$, of p-modes, and amplitudes can be converted into power and energy per mode. Total energy (i.e., kinetic and potential energy) of the $\mathrm{p}$-mode can be given by (Goldreich \& Murray 1994)

$E_{n \ell}=M_{n \ell}\left\langle v_{n \ell}^{2}\right\rangle$,

where the parameter $M_{n . \ell}$ represents the mode mass, which can be computed using the mode inertia, and $\left\langle v_{n \ell}^{2}\right\rangle$ is mean square velocity, the measure of the mode area. It is given by,

$\left\langle v_{n \ell}^{2}\right\rangle=\frac{\pi}{2} A_{n \ell} \Gamma_{n \ell}$

where $A_{n \ell}$ and $\Gamma_{n \ell}$ are mode amplitude and mode width, respectively, obtained from the ring fitting (see Eq. (4)). This expression for the p-mode energy (Eq. (5)) has been used previously to study solar cyclic variation of the p-mode energy of globalmodes e.g., Komm et al. (2000b). The expression for the mean square velocity is different from the expression given in Komm et al. (2000b), who used the correction factor $C_{\text {vis }}=3.33$ for the reduced visibility due to leakage (Hill \& Howe 1998). But for the local modes, $C_{\text {vis }}=1$. We also note that the mode widths computed with the ring-diagram analysis are larger than the actual values and hence the estimated mode energy will be higher. Furthermore, there are many systematic effects on p-mode parameters obtained with the ring-diagram analysis that make it difficult to determine the absolute mode parameters of a given region. Most of these systematic effects can be eliminated, however, by studying the differences in the fitted parameters between different regions with the same observing geometry. Therefore, we analyse mode parameters in ARs and corresponding QRs at same latitude, but at different Carrington longitude. The relative value of the mode energy, using Eqs. (5) and (6), between an AR and corresponding $\mathrm{QR}$ is given by

$\frac{\delta E}{E}=\frac{\left(E_{n \ell}\right)_{\mathrm{AR}}-\left(E_{n \ell}\right)_{\mathrm{QR}}}{\left(E_{n \ell}\right)_{\mathrm{QR}}}=\frac{\left(A_{n} \Gamma_{n \ell}\right)_{\mathrm{AR}}-\left(A_{n \ell} \Gamma_{n \ell}\right)_{\mathrm{QR}}}{\left(A_{n} \Gamma_{n \ell}\right)_{\mathrm{QR}}}$.

This expression for the mode energy is free from mode mass, and hence we do not require mode inertia. Similarly, we analysed fractional differences for other parameters, that is, mode amplitude $(\delta A / A)$, mode width $(\delta \Gamma / \Gamma)$, and background power $\left(\delta b_{0} / b_{0}\right)$ of flaring and dormant ARs along with their corresponding QRs.

\subsection{Magnetic and flare-activity indices}

The MAI (denoted as $B$ ) for every data set of ARs and QRs was calculated from the 96 min averaged MDI magnetograms. Every 
R. A. Maurya et al.: Variations of p-mode amplitude, width, and energy in active regions
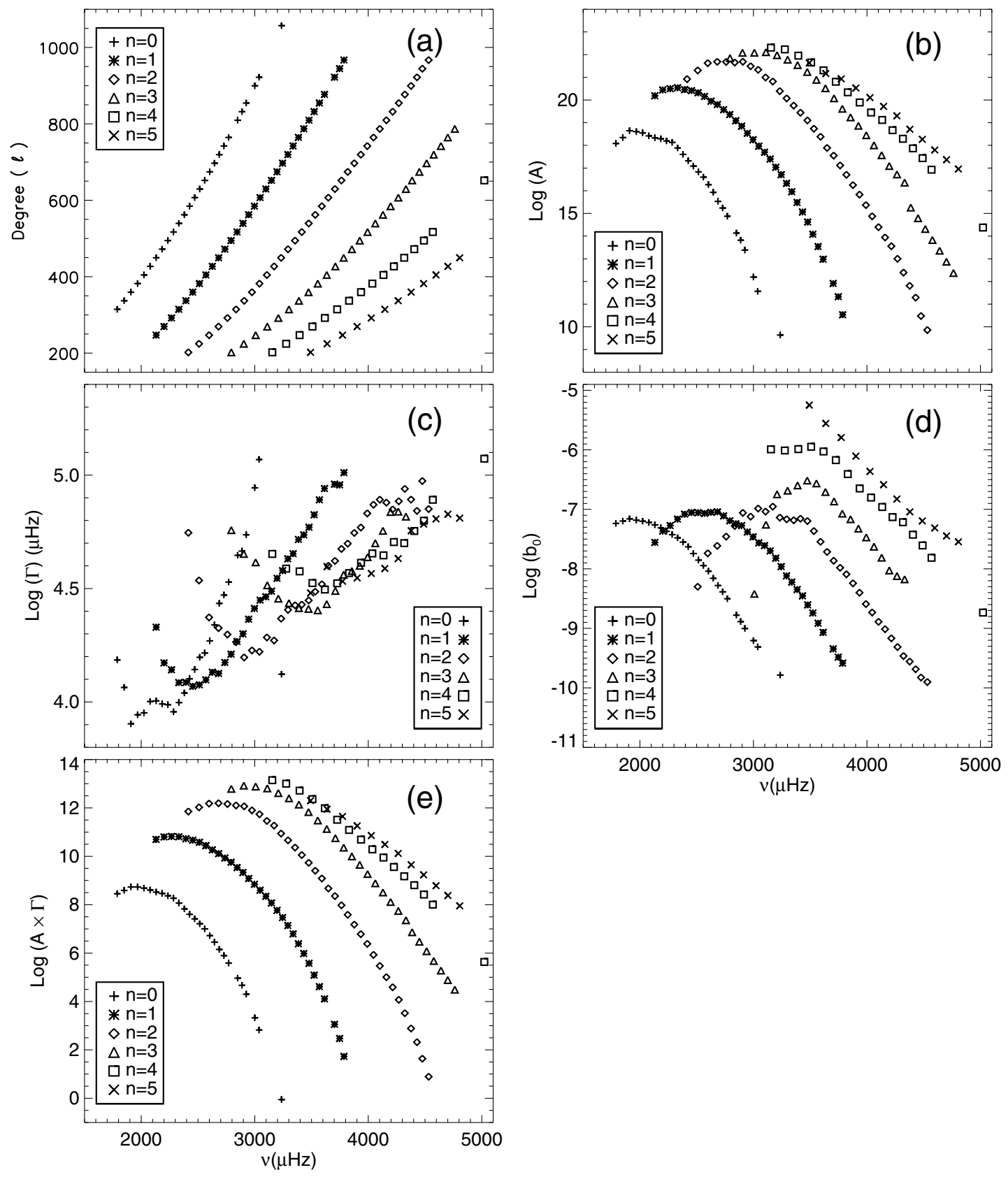

Fig. 2. p-mode parameters obtained for AR NOAA 10649 on 17 July 2004: a) harmonic degree $\ell$; b) mode amplitude $A$; c) mode width $\Gamma$; d) background power $b_{0}$; and e) mode area $A \times \Gamma$ as a function of frequency for radial orders $n=0, \ldots, 5$.

magnetogram was tracked and remapped in the same manner as the Dopplergrams for the ring-diagram analysis. Then areas $\left(16^{\circ} \times 16^{\circ}\right)$ of the ring patches were extracted from the full-disc images. Then, the MAI, $B$, was computed by taking the absolute average over the patches. Since the MDI 96 min averaged magnetograms were obtained from both one- and five-minute magnetograms, we set zero to the pixels with values lower than $35 \mathrm{G}$. Mathematically, the MAI of an AR can be written as

$B=\frac{1}{N_{2}} \sum_{i=1}^{N_{2}}\left(\frac{1}{N_{1}} \sum_{|b|>b_{1}}|b|\right)$,

where $b_{1}$ is the lowest threshold $(=35 \mathrm{G}), N_{1}$ is the number of pixels with $|b|>b_{1}, N_{2}$ is the number of magnetograms observed during the ring-diagram data cube. Thus, the values of $B$ for our samples of flaring and dormant ARs varies in the ranges of 37-308 G and 15-90 G, respectively, while for the QRs it ranges between $1-12 \mathrm{G}$.

A measure of the flare-activity of an AR, the X-ray flare index (say, FI), was calculated by multiplying the GOES X-ray flux with the flare duration and then summing the contributions of all the flares that occurred during the $1664 \mathrm{~min}$ of the data cube for the given AR. The flare index of an AR thus can be written as

$\mathrm{FI}=\sum_{i=1}^{N} F_{i} \delta t_{i}$,

where $F_{i}$ is the GOES X-ray flux for $i^{\text {th }}$ flare event, $\delta t_{i}$ is the time duration of the same event, and $N$ is the total number of flare events that occurred in the time period of the data cube. GOES 


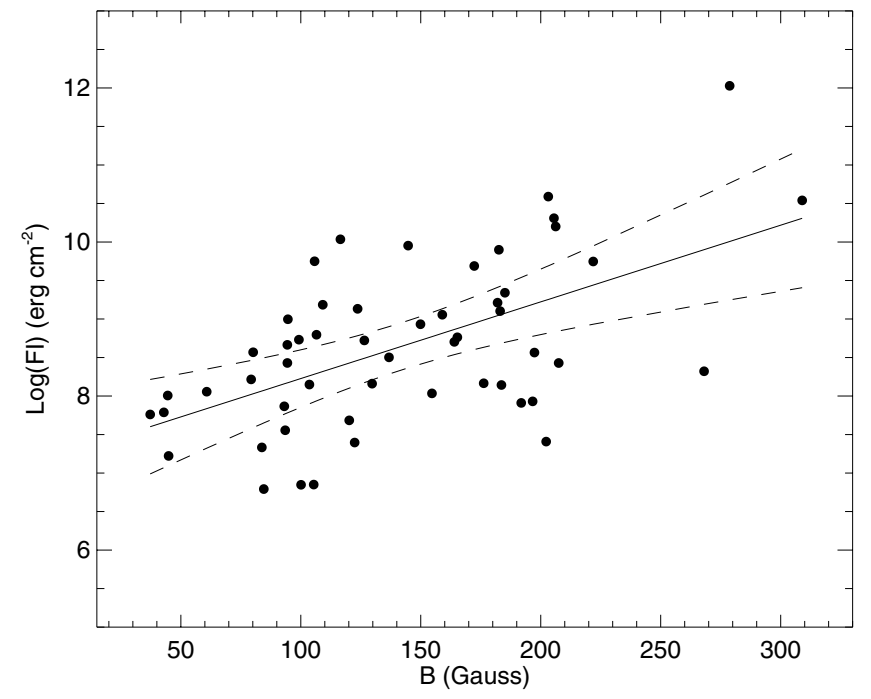

Fig. 3. Relation between magnetic-activity index $(B)$ and flare-activity index (FI) of flaring ARs. Filled circles represent flaring ARs of our sample. The solid line shows the linear regression line, while the dashed lines around it correspond to the $95 \%$ confidence levels of the linear fit.

Table 1. GOES X-class flares and associated energy fluxes.

\begin{tabular}{lc}
\hline \hline X-class & $\begin{array}{c}\text { GOES X-ray Flux } \\
F\left(\mathrm{erg} \mathrm{s}^{-1} \mathrm{~cm}^{-2}\right)\end{array}$ \\
\hline $\mathrm{Xn}$ & $n \times 10^{-1}$ \\
$\mathrm{Mn}$ & $n \times 10^{-2}$ \\
$\mathrm{Cn}$ & $n \times 10^{-3}$ \\
$\mathrm{Bn}$ & $n \times 10^{-4}$ \\
$\mathrm{An}$ & $n \times 10^{-5}$ \\
\hline
\end{tabular}

X-ray fluxes for different flare classes are listed in Table 1. Note that here we only considered the flares of $\mathrm{C}, \mathrm{M}$, and $\mathrm{X}$ classes.

Thus the flare-activity index computed for the flaring ARs is found to range from $8.9 \times 10^{2}$ for moderately active ARs to $1.6 \times 10^{5} \mathrm{erg} \mathrm{cm}^{-2}$ for very productive ARs. Figure 3 shows the relation between the magnetic-activity and flare-activity indices of our sample of flaring ARs. The straight line fit through the data points shows that the flaring-activity index increases with the MAI of ARs. The 95\% confidence levels (dotted curves) of the fitting further validate this linear relation between FI and $B$.

\subsection{Mode corrections}

For a given multiplet $(n, \ell)$, mode parameters change from region to region and with time. These changes are the combined effects of foreshortening, duty cycle, magnetic-activity, flareactivity, and measurement uncertainties. To study the activityrelated changes in oscillation modes, we need to analyse and correct for the other effects.

Here the duty cycle is the filling of the observed Dopplergrams in the data cube used for p-mode computation with the ring-diagram analysis. In our data samples, the duty cycle varies in the range $70-100 \%$. However, most of the data cubes have duty cycles $>80 \%$. Note that we analysed the relative variations in the mode parameters of ARs and corresponding QRs (e.g., Eq. (7)) that have the same duty cycles.

The Dopplergrams are significantly affected by foreshortening effects, i.e., as we go away from the disc centre, we measure only the cosine component of the vertical displacement. Another effect of foreshortening in mode parameters is caused by the fact that the spatial resolution in Dopplergrams decreases as we observe increasingly towards the limb. For example, a pixel which has a spatial resolution of $\mathrm{d} x$ at the disc centre, now images a horizontal distance on the Sun of $\mathrm{d} x / \rho$, where $\rho=\cos \Lambda \sin \Theta$. This reduces the spatial resolution measured on the Sun in the centre-to-limb direction, and hence leads to systematic errors.

Since we aim to compare the mode parameters in different ARs, we analysed only the common modes among them. A mode is called common if all the data sets of ARs and QRs have same radial order $(n)$ and degree $(\ell)$. We found a total of 98 common modes in our sample of ARs and QRs, while the total number of fitted modes ranges between 125-173. Figure 4 shows examples of changes in common mode-parameters of all the dormant ARs and QRs with the central meridian distance $(\lambda)$, the duty cycle $(f)$, and the MAI $(B)$.

One can infer from Fig. 4 that the mode amplitude $(A)$ and background power $\left(b_{0}\right)$ are more affected with distance from the disc centre than the mode width $(\Gamma)$. The mode amplitude and background power decrease with increasing distance from the disc center, while the mode width shows an opposite relation. The variations in mode parameters with $\lambda$ are best modelled by the quadratic function in $\lambda$ (see the quadratic fit in Fig. 4). The variations in background power with distance from the disc centre are stronger than the changes with the MAI. We also note that the measured longitude and latitude are affected by the position angle ( $P$ angle) and $B_{0}$ angle of the Sun.

Changes in mode parameters with duty cycles are shown in Fig. 4 (middle columns). The mode amplitude increases with increasing duty cycle in the data cubes, while the mode width and background power show the opposite trend. However, it is evident that the duty-cycle related variations in mode parameters are much weaker than the variations due to the foreshortening and magnetic-activity indices.

We modelled the effects of foreshortening and duty cycles on a common mode-parameter $\left(\mathcal{P}_{n, \ell}\right)$ of all the ARs and corresponding QRs by a multiple non-linear regression as a function of the central meridian distance $(\lambda)$ and latitude $(\theta)$ of the centres of ring patches, position angle $(P)$, and B-angle $\left(B_{0}\right)$ of the Sun, and the duty cycle $(f)$ of data cubes,

$$
\begin{aligned}
\mathcal{P}_{n, \ell}\left(\lambda, \theta, \lambda_{0}, \theta_{0}, f\right)= & C_{0}+C_{1} \lambda+C_{2} \lambda^{2}+C_{3} \theta+C_{4} \theta^{2} \\
& +C_{5} P+C_{6} P^{2}+C_{7} B_{0}+C_{8} B_{0}^{2} \\
& +C_{9} f
\end{aligned}
$$

where $C_{0}, C_{1}, \ldots, C_{9}$ are regression coefficients. We repeated this analysis for all the common pairs $(n, \ell)$ of the ARs and QRs.

\subsubsection{Effects of the foreshortening on the mode parameters}

Figure 5 shows the multiple non-linear regression coefficients $\left(C_{1}, C_{2}\right.$ and $\left.C_{9}\right)$, normalized by the intercept $C_{0}$, for the common modes in all ARs and QRs as a function of the harmonic degree, $\ell$. The corresponding Pearson correlation coefficients are shown in Fig. 6. The coefficients $C_{2}$ of the second-order term for all the mode parameters are lower than the coefficients $C_{1}$ of the first-order term by one order of magnitude. But the correlation coefficients $R_{2}$ for all mode parameters are twice as high as the coefficients $R_{1}$, suggesting a better association between the mode parameters and the quadratic term in $\lambda$. Two coefficients $C_{1}$ and $C_{2}$ are negative for the mode parameters $A$ and $b_{0}$ for all the harmonic degrees. For the mode width $\Gamma$, values of $C_{1}$ are positive for most of the modes with radial order $n=0,1$ 
R. A. Maurya et al.: Variations of p-mode amplitude, width, and energy in active regions
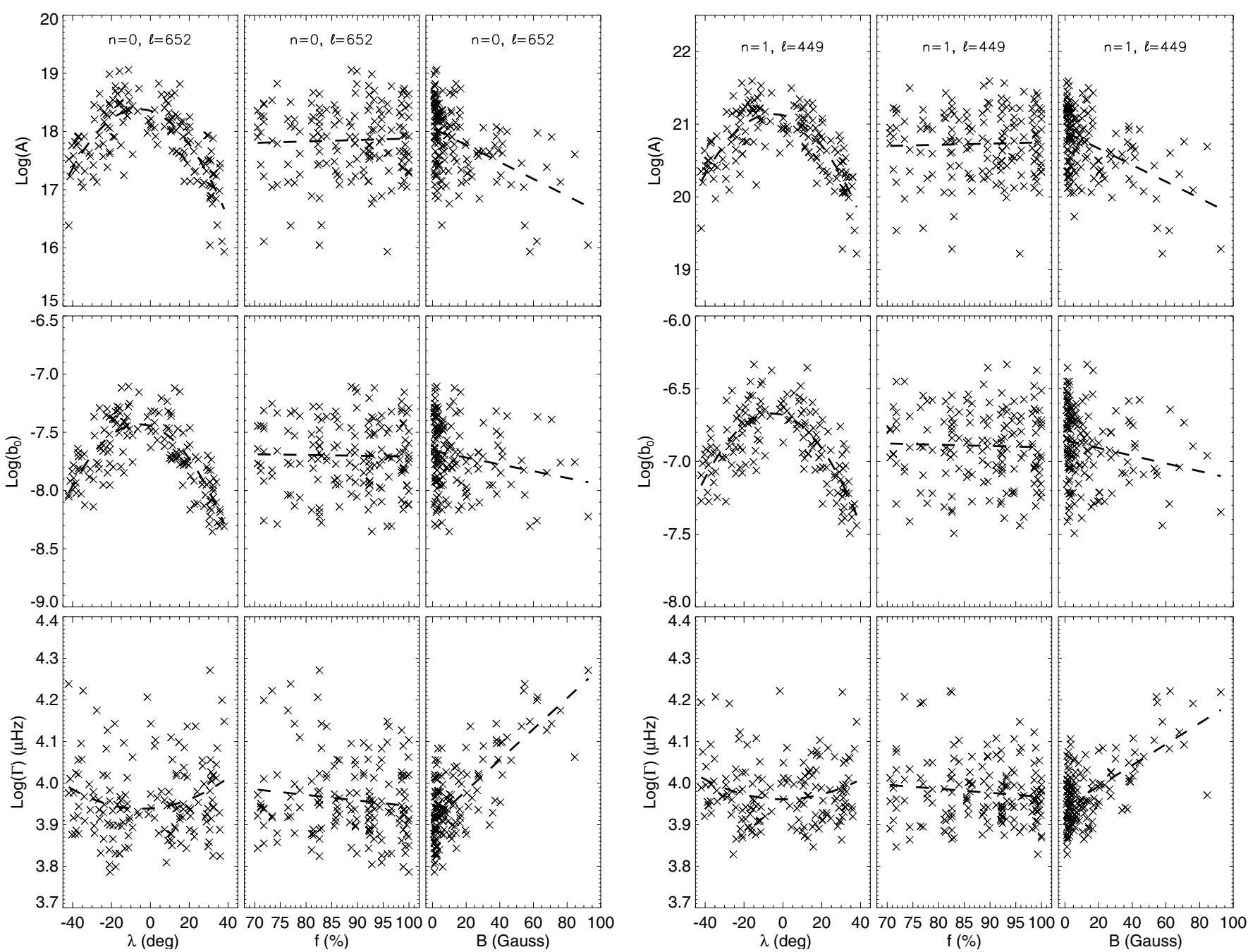

Fig. 4. Mode parameters, amplitude $A$ (top row), background power $b_{0}$ (middle row), and width $\Gamma$ (bottom row) for two modes ( $n=0, \ell=652$ (left three columns); $n=1, \ell=449$ (right three columns)) for dormant ARs and QRs as a function of the central meridian distance $\lambda$ of the region centres (left), duty cycle $f$ (middle), and magnetic-activity index $B$ (right). Dashed curves show the quadratic fit (in $\lambda$ ) and the linear fit (in $f$ and $B$ ) through the mode parameters.

and negative for $n=2,3$. Moreover, the magnitude of the coefficients for $A$ and $b_{0}$ is higher than that for $\Gamma$ for all harmonic degrees $\ell$. This shows that the mode amplitude and background power decrease rapidly while the mode width increases slowly with increasing distance from the disc centre. Similar changes are also seen in the corresponding plots for correlation coefficients $R_{1}$ and $R_{2}$ (Fig. 6).

Regression coefficients $C_{1}$ and $C_{2}$ for $A$ increase in magnitude with increasing harmonic degree $(\ell)$, but the mode width does show a significant relation with $\ell$ in regression as well as in correlation coefficients. Figure 6 shows a stronger anticorrelation between the quadratic term in the mode parameter $A$ and in $\lambda$ than that of the linear term. There is a weak positive correlation between the distance from the disc centre and the mode width. But the magnitudes of the correlation are very weak, $<0.10$. Regression coefficients $C_{1}$ and $C_{2}$ for background power decrease with increasing $\ell$; this is also evident from the significant anti-correlation between $b_{0}$ and $\lambda$. The correlation coefficients for the background power changes with $\ell$ and radial or$\operatorname{der} n$. Thus we find that the background power is predominantly a function of harmonic degree $(\ell)$, decreasing with increasing $\ell$.

Similarly, the effects of latitude $(\theta), \mathrm{P}$ angle $(P)$ and $\mathrm{B}$ angle $\left(B_{0}\right)$ on the mode parameters can be illustrated. The foreshortening-corrected mode parameters $\left(\mathcal{P}_{n, \ell}^{c}\right)$ were obtained by subtracting the first- and second-order terms (see Eq. (10)) from the common mode-parameters $\left(\mathcal{P}_{n, \ell}\right)$. The foreshortening effects on the p-mode amplitude and width have also been studied previously (e.g., Howe et al. 2004). Our results support these results.

\subsubsection{Effects of duty cycle on the mode parameters}

The duty cycle coefficient $C_{9}$ for the mode amplitude $A$ decreases with increasing $\ell$ (Figs. 5). It is positive for almost all modes at lower degree $(\ell \lesssim 600)$. In the intermediate range of degree, a few modes have negative $C_{9}$, but with lower magnitudes. The positive value of the coefficient $C_{9}$ shows that the mode amplitude increases with increasing duty cycle, while negative $C_{9}$ correspond to the opposite relation. The increase of $A$ with $f$ is obvious from the increase in signal samples (or number of observed Dopplergrams) in the data cubes, but the reverse is not clear. This may be attributed to the distribution of observed Doppler frames in the data cubes. However, the magnitude of the correlation coefficients $C_{9}$ is very low for degree $\ell>450$.

The regression coefficients $C_{9}$ for the mode width and background power is negative at all degrees (Fig. 5). The magnitude 
A

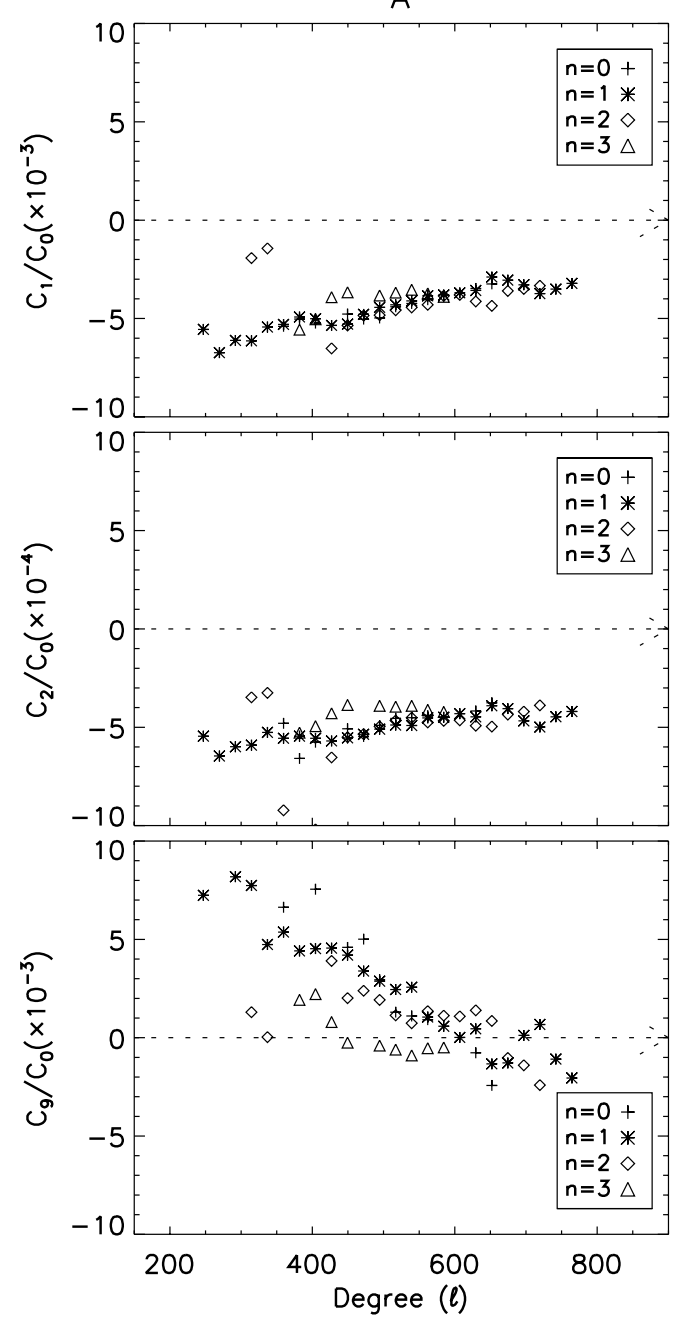

$\Gamma$

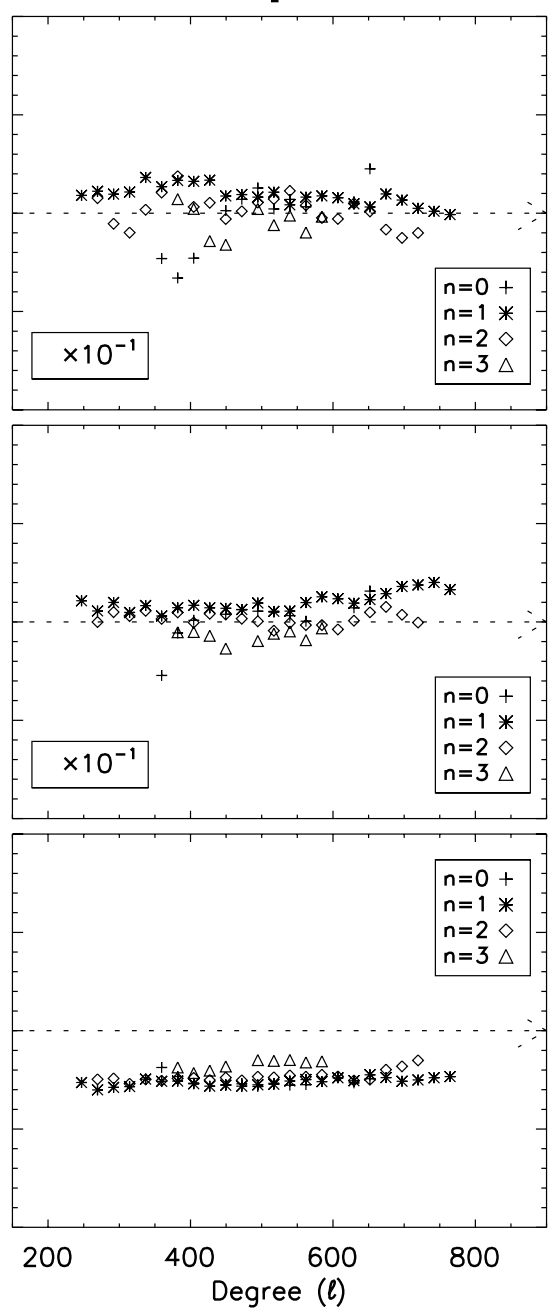

$b_{0}$

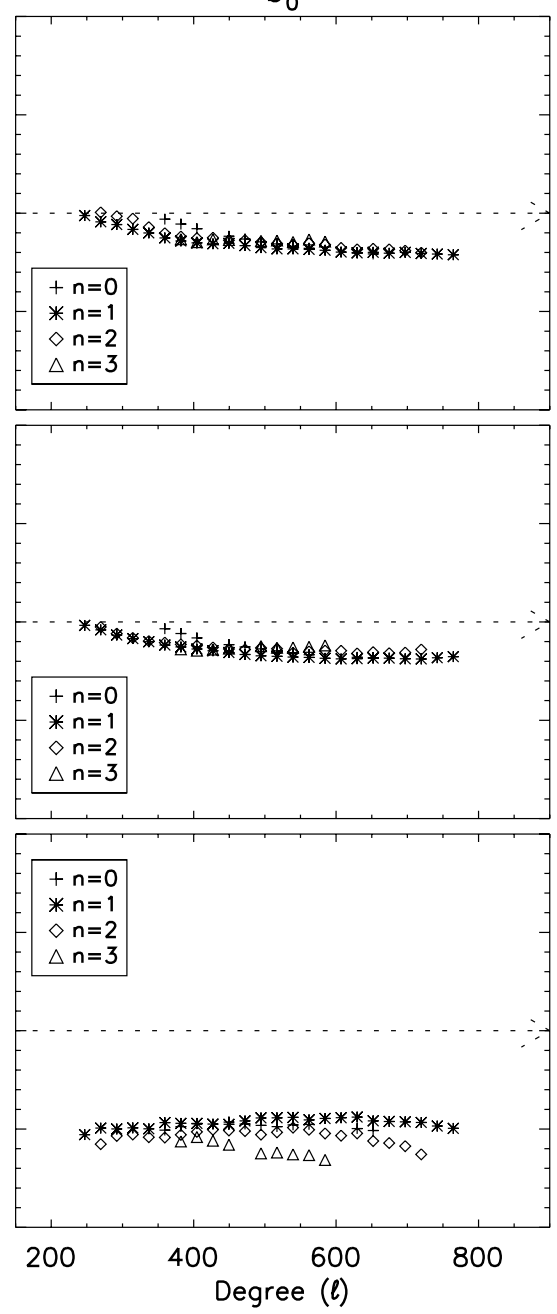

Fig. 5. Multiple non-linear regression coefficients, $C_{1}$ (top row), $C_{2}$ (middle row), and $C_{9}$ (bottom row), normalized with intercept, $C_{0}$, for the mode parameters, A (left column), $\Gamma$ (middle column), and $b_{0}$ (right column) as a function of harmonic degree, $\ell$. Corresponding correlation coefficients are shown in Fig. 6.

of $C_{9}$ is lowest at lower $\ell$ and decreases with increasing $\ell$. Figure 6 also shows the anti-correlation between the mode width and the duty cycle. But the anti-correlation coefficients decrease with increasing harmonic degree $\ell$. The above values of $C_{9}$ and $R_{9}$ show that the mode width and background power decrease with increasing duty cycle.

\section{Results and discussions}

\subsection{Variation in p-mode parameters with magnetic-activity index}

To analyse the variation in mode parameters with MAI $(B)$ of flaring and dormant ARs, we applied the mode corrections for foreshortening and duty cycle to all the mode parameters of all the ARs and QRs.

Figure 7 shows the averaged mode parameters in QRs, dormant ARs, and flaring ARs as a function of frequency. Evidently, the average mode amplitudes in QRs are larger than those in dormant and flaring ARs for all radial orders. Flaring ARs, possessing the highest $B$ values, show the smallest average mode amplitude in all radial orders. This is obviously due to the stronger mode-power suppression in ARs, which have a stronger MAI $(B)$ than the QRs. The average background power for $n \leq 2$ in ARs and QRs varies with $B$ similar to the average mode amplitude. For $n \geq 3$, the background power is strongest in flaring ARs and weakest in QRs. The average mode widths are largest in flaring ARs and smallest in QRs for all radial orders, which shows lifetimes of modes in flaring ARs shorter than in dormant ARs and QRs. The mode area $(A \times \Gamma)$ of ARs and QRs shows similar trend as the mode amplitude.

Figures 8 and 9 show the frequency averages of the fractional difference in mode parameters as a function of the MAI difference ( $\left.\delta B=B_{\mathrm{AR}}-B_{\mathrm{QR}}\right)$ between ARs and corresponding QRs for flaring and dormant ARs in three frequency bands, as indicated. The coefficients of linear regression and Pearson correlation between the frequency-averaged fractional mode differences in the three frequency bands and $\delta B$ are given in Table 2. Averaging the mode parameters in these frequency bands would somewhat hide the frequency-dependent properties of the modes.

Previous statistical studies of several ARs showed that mode amplitude and width are linearly related (e.g., Howe et al. 2004; Rabello-Soares et al. 2008). Therefore, to find the harmonic degree and frequency-dependent relation between mode parameters and $\delta B$, we fitted the fractional mode differences $(\mathcal{R})$ and $\delta B$ with linear regression, $\mathcal{R}_{n, \ell}(\delta B)=\alpha_{0}+\alpha_{1}(\delta B)$. The slope $\left(\alpha_{1}\right)$ of the linear regression then corresponds to the mode parameter variation per Gauss, hereafter the "parameter variation rate". 
R. A. Maurya et al.: Variations of p-mode amplitude, width, and energy in active regions
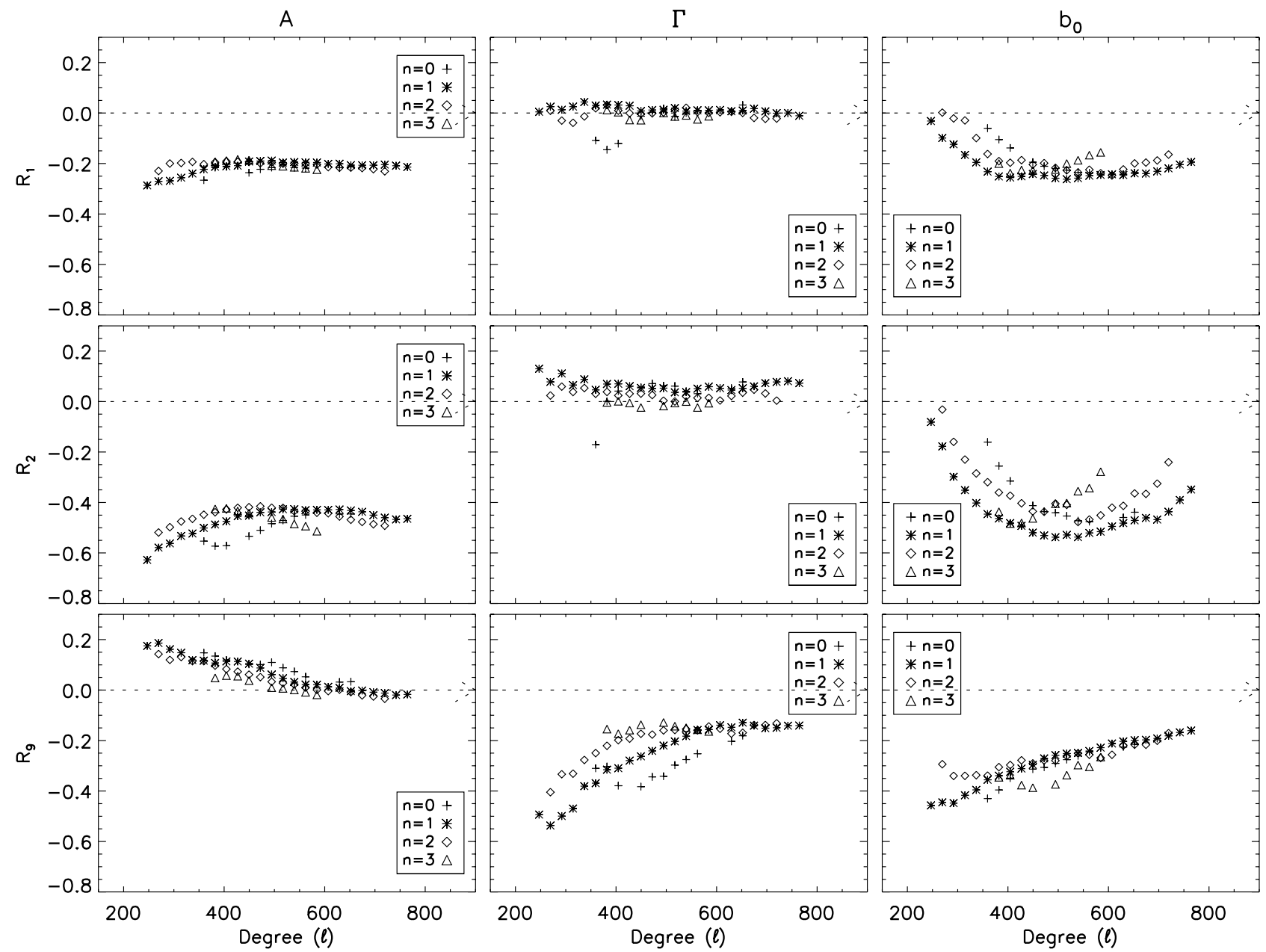

Fig. 6. Correlation coefficients, $R_{1}$ (top row), $R_{2}$ (middle row), and $R_{9}$ (bottom row) corresponding to first and second-order terms in central meridian distance $\lambda$ (see Eq. (10)), respectively, for the mode parameters, $A$ (left column), $\Gamma$ (middle column), and $b_{0}$ (right column) as a function of harmonic degree, $\ell$.

Table 2. Linear regression $(y=a+b \delta B)$ and Pearson correlation coefficients $(r)$ obtained from the fractional mode differences $(y)$ in three frequency bands and magnetic-activity indices $(\delta B)$.

\begin{tabular}{lc|ccc|ccc}
\hline \hline \multirow{2}{*}{$\begin{array}{l}\text { Mode } \\
\text { Parameters }\end{array}$} & \multicolumn{3}{|c|}{ Flaring ARs } & \multicolumn{3}{c}{ Dormant ARs } \\
\cline { 3 - 8 } & $(\mu H z)$ & \multicolumn{3}{|c|}{$\left(\times 10^{-3}\right.$ Gauss $\left.^{-1}\right)$} & & \multicolumn{3}{c}{$\left(\times 10^{-3} \mathrm{Gauss}^{-1}\right)$} \\
\hline \multirow{2}{*}{$\delta A / A$} & $1800-2799$ & -0.16 & $-1.60 \pm 0.18$ & -0.78 & -0.03 & $-3.27 \pm 0.63$ & -0.60 \\
& $2800-3799$ & -0.33 & $-1.24 \pm 0.29$ & -0.52 & -0.08 & $-5.20 \pm 0.97$ & -0.61 \\
& $3800-4800$ & -0.29 & $-1.15 \pm 0.31$ & -0.46 & -0.04 & $-5.13 \pm 1.23$ & -0.51 \\
\hline \multirow{2}{*}{$\delta b_{0} / b_{0}$} & $1800-2799$ & -0.01 & $0.00 \pm 0.48$ & 0.00 & 0.05 & $-2.05 \pm 1.05$ & -0.27 \\
& $2800-3799$ & -0.13 & $-0.10 \pm 0.24$ & -0.06 & -0.02 & $-1.45 \pm 0.40$ & -0.46 \\
& $3800-4800$ & -0.11 & $0.46 \pm 0.31$ & 0.20 & -0.04 & $-0.85 \pm 0.53$ & -0.22 \\
\hline \multirow{3}{*}{$\delta \Gamma / \Gamma$} & $1800-2799$ & 0.05 & $1.01 \pm 0.14$ & 0.71 & 0.00 & $1.40 \pm 0.21$ & 0.69 \\
& $2800-3799$ & 0.18 & $1.57 \pm 0.26$ & 0.65 & 0.02 & $3.89 \pm 0.37$ & 0.83 \\
& $3800-4800$ & 0.16 & $1.25 \pm 0.24$ & 0.58 & 0.02 & $3.63 \pm 0.47$ & 0.74 \\
\hline \multirow{3}{*}{$\delta E / E$} & $1800-2799$ & -0.10 & $-1.33 \pm 0.19$ & -0.71 & -0.03 & $-2.33 \pm 0.56$ & -0.51 \\
& $2800-3799$ & -0.19 & $-0.94 \pm 0.38$ & -0.33 & -0.05 & $-3.06 \pm 0.95$ & -0.42 \\
& $3800-4800$ & -0.15 & $-0.90 \pm 0.39$ & -0.31 & -0.02 & $-2.88 \pm 1.18$ & -0.33 \\
\hline
\end{tabular}

Notes. The mode parameters are corrected for the foreshortening and the duty cycles.

The coefficients $\alpha_{1}$ of the linear regression for different mode parameters are shown in Figs. 10 and 11 as a function of harmonic degree $(\ell)$ and frequency $(v)$, respectively. The constant term $\alpha_{0}$ has no intrinsic meaning and is not illustrated. In the following, we discuss various mode parameters.

\subsubsection{Mode amplitude}

Figure 8 (left two columns) shows the frequency-averaged fractional differences in mode amplitudes $(\delta A / A)$ in three frequency bands. For both flaring and dormant ARs, $\delta A / A$ are below zero, 

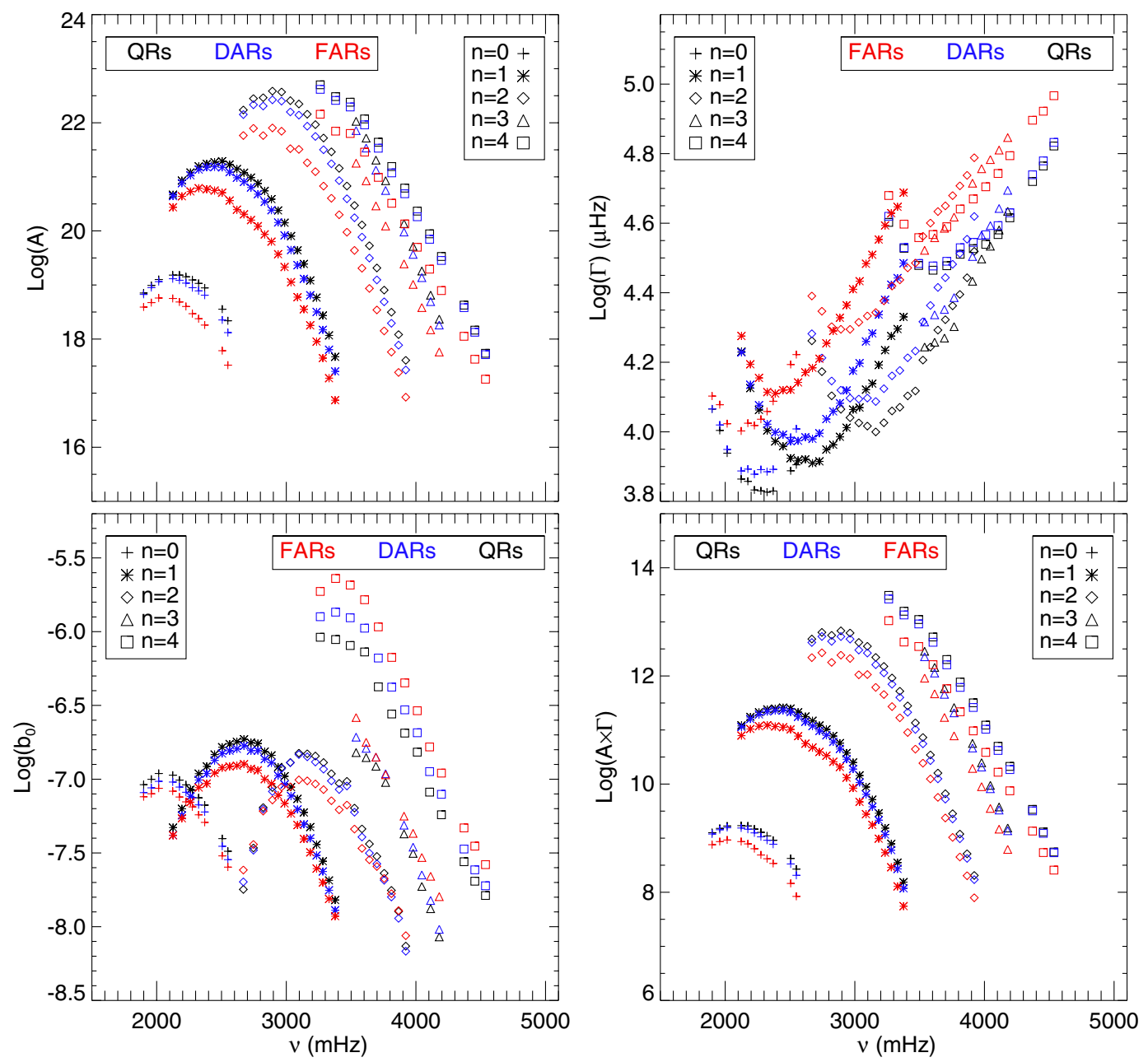

Fig. 7. Average common mode-parameters in QRs (black), dormant ARs (DARs; blue), and flaring ARs (FARs; red) for different radial orders as a function of mode frequency. Note that mode parameters are corrected for foreshortening and duty cycle before averaging. (This figure is available in color in electronic form.)

that is, negative. However, they are more negative for flaring ARs than for dormant ARs. The amplitude difference decreases with increasing $\delta B$, which is further confirmed by the significant correlation coefficients ( $r$ ) between $\delta A / A$ and $\delta B$ (see Table 2). The magnitude of the correlation coefficients is larger in the lower frequency band than in higher frequency bands for flaring ARs. In the flaring ARs, the power suppression per unit Gauss is stronger than in dormant ARs, as is evident from the linear regression slope $b$ (see Table 2). Furthermore, in the five-minute band of dormant ARs, we infer a stronger suppression than in the lower and higher frequency bands.

The amplitude variations for both flaring and dormant ARs are well correlated with $\delta B$, as the evident from the magnitude of Pearson correlation coefficients (see Figs. 10 and 11). This confirms the linear relation between amplitude variation and $B$ (Rabello-Soares et al. 2008).

Figures 10 and 11 show changes in the linear regression coefficient $\alpha_{1}$ for the mode amplitude (i.e., rate of change of $\delta A / A$ per unit Gauss) as a function of harmonic degree $(\ell)$ and frequency $(v)$, respectively. For the flaring ARs, the regression coefficients $\alpha_{1}$ increase slowly with harmonic degree $\ell$. The correlation coefficients also decrease in magnitude with increasing $\ell$. Furthermore, the correlation coefficients for both flaring and dormant ARs change with harmonic degree. For the dormant ARs, the magnitude of the correlation coefficients for different radial orders first decreases with harmonic degree $(\ell)$, becomes smallest at $\ell$ in the range $\sim 450-600$, and then increases again. This is consistent with the results Bogdan et al. (1993), who reported maximum absorption around wavenumber $k \approx 0.8 \mathrm{Mm}^{-1}$ or $\ell \approx 550$. This value of $\ell$ approximately agrees with our values, where we find strongest suppression of power for $n=1,2$, and 3 modes. For the flaring ARs, the correlation coefficients monotonically increase with degree, except for a few modes at lower degree, for all the radial orders. The significant correlation coefficients further support the relationships between $\delta A / A$ and $\delta B$ of the flaring and dormant ARs.

Figure 11 (first row from top) shows that the amplitude change in dormant ARs with frequency is not monotonic. The magnitude of the regression coefficient $\alpha_{1}$ of the dormant ARs decreases with frequency and becomes smallest in the fiveminute band, then decreases. For flaring ARs, $\alpha_{1}$ does not show a significant frequency dependence.

The amplitude decrease rate (i.e., $\alpha_{1}$ ) for dormant ARs increases with frequency and peaks around $3.0 \mathrm{mHz}$ and $3.5 \mathrm{mHz}$ for the radial orders $n=1$ and $n=2$, respectively. This supports the previous reports (Rajaguru et al. 2001; Howe et al. 2004; Rabello-Soares et al. 2008). For global modes, Komm et al. (2000a) found about 7\% decrease in mode amplitude with the solar cycle. They also found that the strongest variations of $29 \%$ occur in the frequency range of $2.7-3.3 \mathrm{mHz}$. More interestingly, 

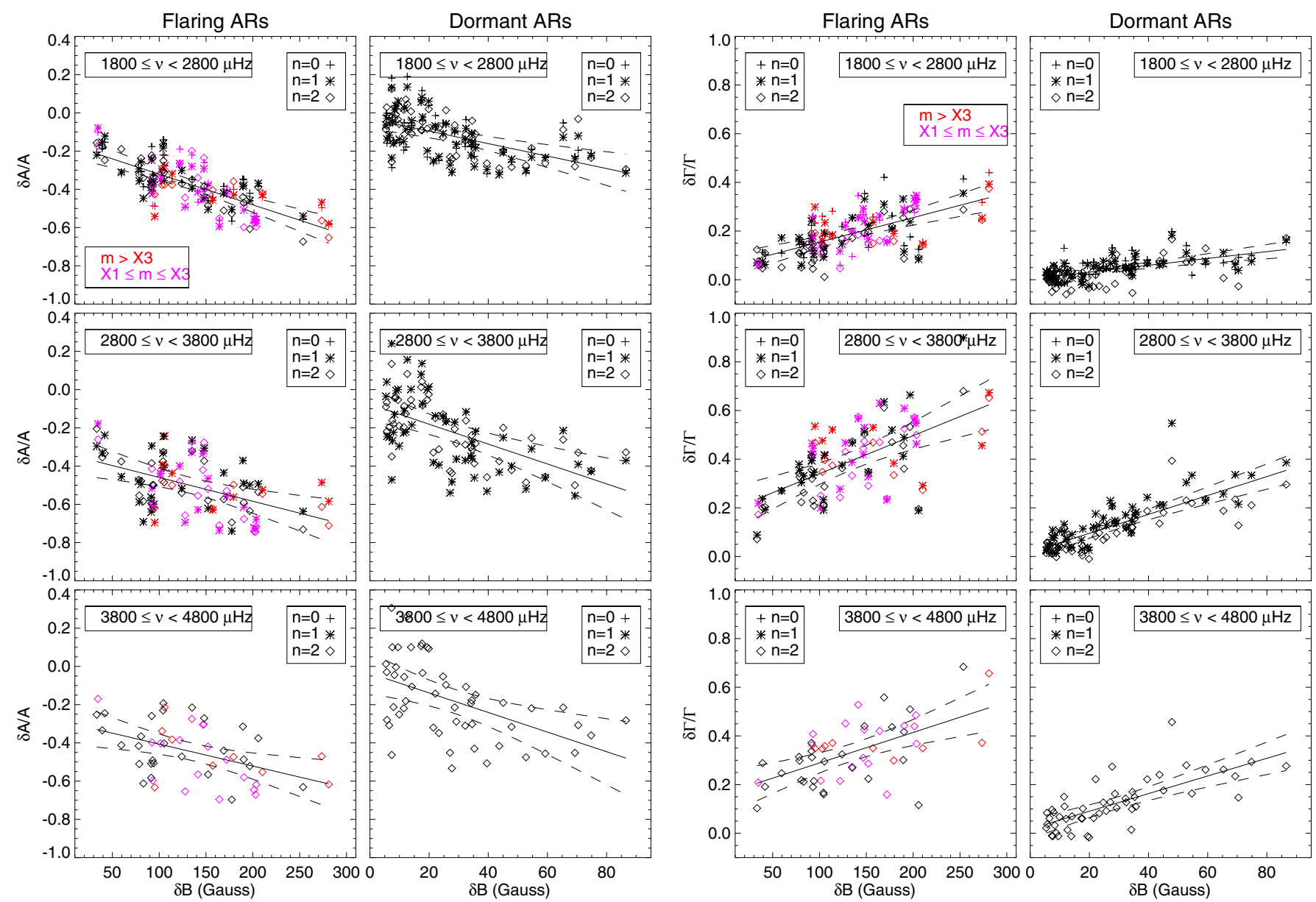

Fig. 8. Frequency averages of the fractional differences in mode amplitude (left two columns) and mode width (right two columns) for flaring (left) and dormant (right) ARs as a function of the magnetic-activity index difference $\left(\delta B=B_{\mathrm{AR}}-B_{\mathrm{QR}}\right)$ of the AR and the corresponding QR. Red and magenta symbols correspond to the ARs flare(s) of magnitude $(m)>X 3$ and $X 1 \leq m \leq X 3$, respectively. The solid lines show the linear regression fit, while dashed curves show $90 \%$ confidence level of the linear fit. Note that the mode parameters are corrected for the foreshortening and duty cycles. (This figure is available in color in electronic form.)

we find that the amplitude variation rate for dormant ARs increases at higher frequencies. The observed increase of the mode absorption as a function of frequency and decrease at higher frequencies have been theoretically reported previously (Jain et al. 2009). Negative values of $\alpha_{1}$ may be attributed to the power absorption (Braun et al. 1987). But it is not clear whether the mode power absorption can be compared with the suppression of power in the sunspot region, because the absorption was calculated for travelling waves, while the modes we analysed are standing waves.

Lower values of the coefficients $\alpha_{1}$ for the flaring ARs than for the dormant ARs may be the combined result of both mode power absorption by sunspots and amplification by flares. However, the absorption effect clearly dominates, as is evident from the negative fractional difference in mode amplitude (Fig. 8).

\subsubsection{Mode width}

The mode width of a peak profile in the power spectrum is related to the imaginary part of the frequency and hence is a measure of mode damping. It is inversely proportional to the lifetime of the mode. The frequency-averaged fractional difference in mode width $(\delta \Gamma / \Gamma)$ for flaring and dormant ARs as a function of MAI difference $(\delta B)$ is shown in Fig. 8 (right two columns). $\delta \Gamma / \Gamma$ of both the flaring and dormant ARs are positive and increase with increasing $\delta B$, employing the decrease in lifetime of modes with $B$. In the five-minute band of both the flaring and dormant ARs, $\delta \Gamma / \Gamma$ increases faster than in lowerand higher-frequency bands. This relation is further confirmed from the high correlation coefficients (see Table 2). But the correlations are better in the five-minute band of the dormant ARs and in the lower-frequency band of flaring ARs. The higher values of the intercept $a>0$ for dormant ARs than flaring ARs show that the mode width is larger in ARs than in QRs. Thus it appears that most modes live longer in QRs than they do in ARs, employing the higher damping in ARs. For dormant ARs, data points are closer to the linear regression line, while for flaring ARs there is a large spread at larger $\delta B$, causing lower correlations. This may be caused by activities in the flaring ARs. Furthermore, the steeper slope in the five-minute band of flaring ARs than in dormant ARs shows that the mode width in flaring ARs decreases more slowly with $\delta B$ than in dormant ARs.

The coefficients $\left(\alpha_{1}\right)$ of the linear regression between the fractional difference in mode width and $\delta B$ are shown in Figs. 10 and 11 (second row from the top) as a function of harmonic degree $(\ell)$ and frequency $(v)$, respectively. The coefficients $\alpha_{1}$ for both dormant and flaring ARs are positive and increase with $\ell$. Its values are lower for flaring ARs than the dormant ARs for all $\ell$ except for a few modes with $\ell<350$. The highest value 

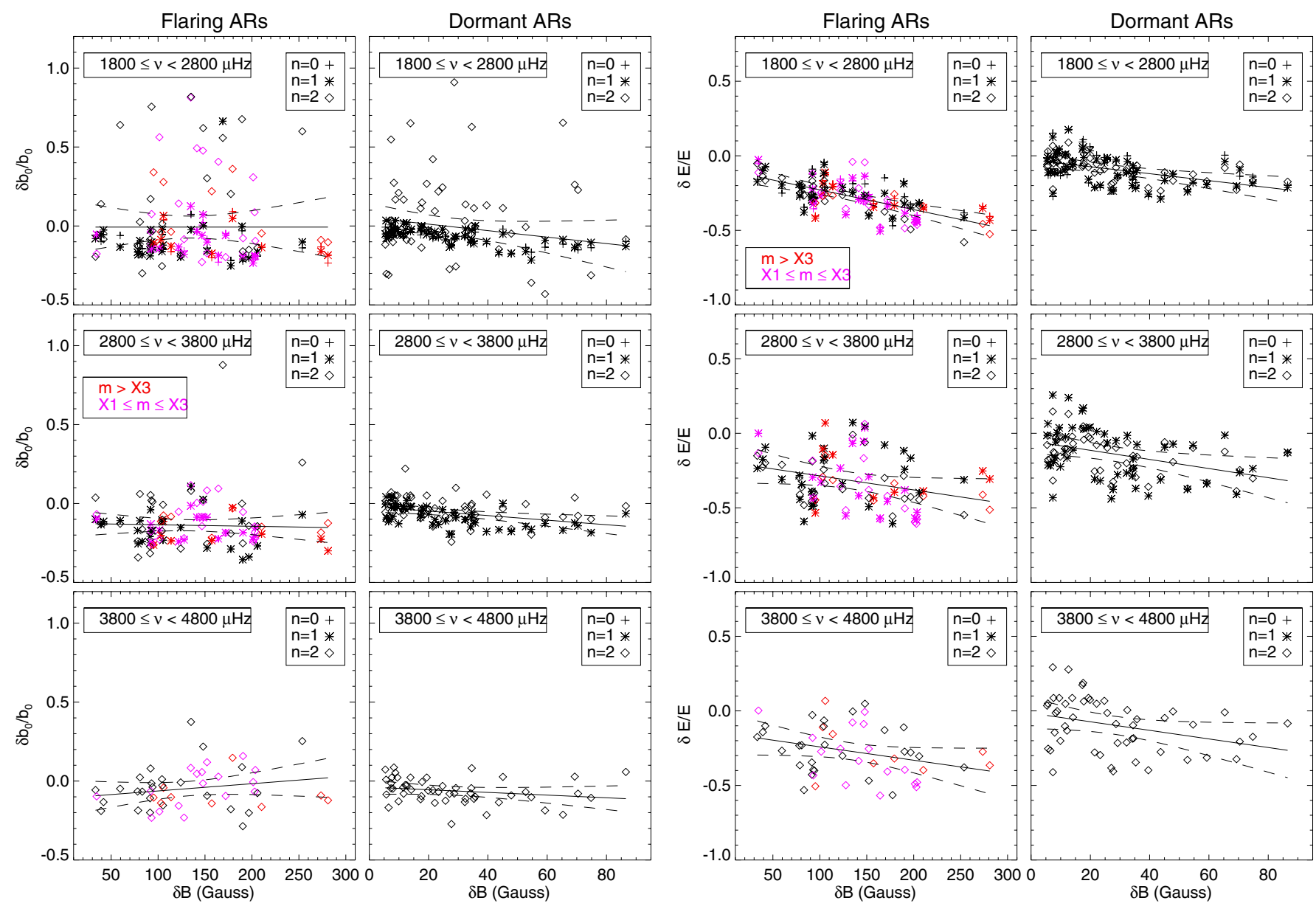

Fig. 9. Similar to Fig. 8, but for the background power (left two columns) and mode energy (right two columns). (This figure is available in color in electronic form.)

of the width variation for flaring and dormant ARs occurs in the range $\sim 1-2$ Gauss $^{-1}$ and $\sim 2-6$ Gauss $^{-1}$, respectively.

Chen et al. (1996), using the absorption of p-mode waves in sunspots as a tool to determine the lifetime of p-modes in the $\ell$ range (200-700), reported that $\mathrm{p}$-mode lifetimes decrease with $\ell$ and frequency $(v)$. The width-variation rate of dormant ARs initially increases with frequency for all radial orders, peaks at different frequencies in the five-minute frequency band, and then decreases (except $n=0$ ). For radial order, $n=0$, the maximum of about 0.0032 Gauss $^{-1}$ occurs around $v \approx 2.6 \mathrm{mHz}$. But note that we did not cover the modes $v>2.6$ for $n=0$. Rajaguru et al. (2001), Howe et al. (2004), and Rabello-Soares et al. (2008) have reported the maximum around $0.005,0.0045$ and $0.003 \mathrm{Gauss}^{-1}$, respectively.

For the global modes, Komm et al. (2000a) reported solar cyclic mode-width variations of about $3 \%$ and the maximum changes of $47 \%$ in the frequency range $2.7-3.5 \mathrm{mHz}$. But they did not find $\ell$ dependence of the solar cycle changes in mode width, presumably because of weaker variations in the average global magnetic fields.

\subsubsection{Background power}

The background power in solar oscillation spectra is not just "noise", but contains physical information that might be important for a better understanding of the dynamics of the solar atmosphere. It has a large component of so-called solar noise, which is the background produced by convective cells. Furthermore, the detection of oscillation modes essentially depends upon the signal-to-noise ratio, therefore, it is important to compare the background noise in the power spectra between the ARs and corresponding QRs. In addition, it should be noted that the mode background power may contain substantial contributions from mode "tails", particularly when the mode is asymmetric because it is not taken into account in the fitting (cf., Eq. (4)).

Figure 9 (left two columns) shows that the relative variation in frequency-averaged background power of dormant ARs decreases slowly with increasing $\delta B$ while it is almost constant for flaring ARs. For most of the ARs, the background power is weaker than their corresponding QRs, which confirms previous reports (Rajaguru et al. 2001). The reduction is expected because the magnetic field is known to suppress convection, which is the main source of background in power spectra. But the fractional difference in background power at lower-frequency band is larger than zero for a few modes with radial order, for example, $n=2$ (see Fig. 9). The correlation between $\delta b_{0} / b_{0}$ and $\delta B$ is poor or non-existent in flaring ARs, while there is significant anti-correlation for dormant ARs (see Table 2). The poor correlation coefficient for flaring ARs may be caused by mode suppression due to sunspots and amplification due to flares.

Komm et al. (2000a) found no significant variation in background power with solar cycle in the global modes. The expected variation in background power with solar cycle in their analysis may be due to the weak increase in the average magnetic field with the solar cycle. Moreover, the global mode analysis is 
R. A. Maurya et al.: Variations of p-mode amplitude, width, and energy in active regions
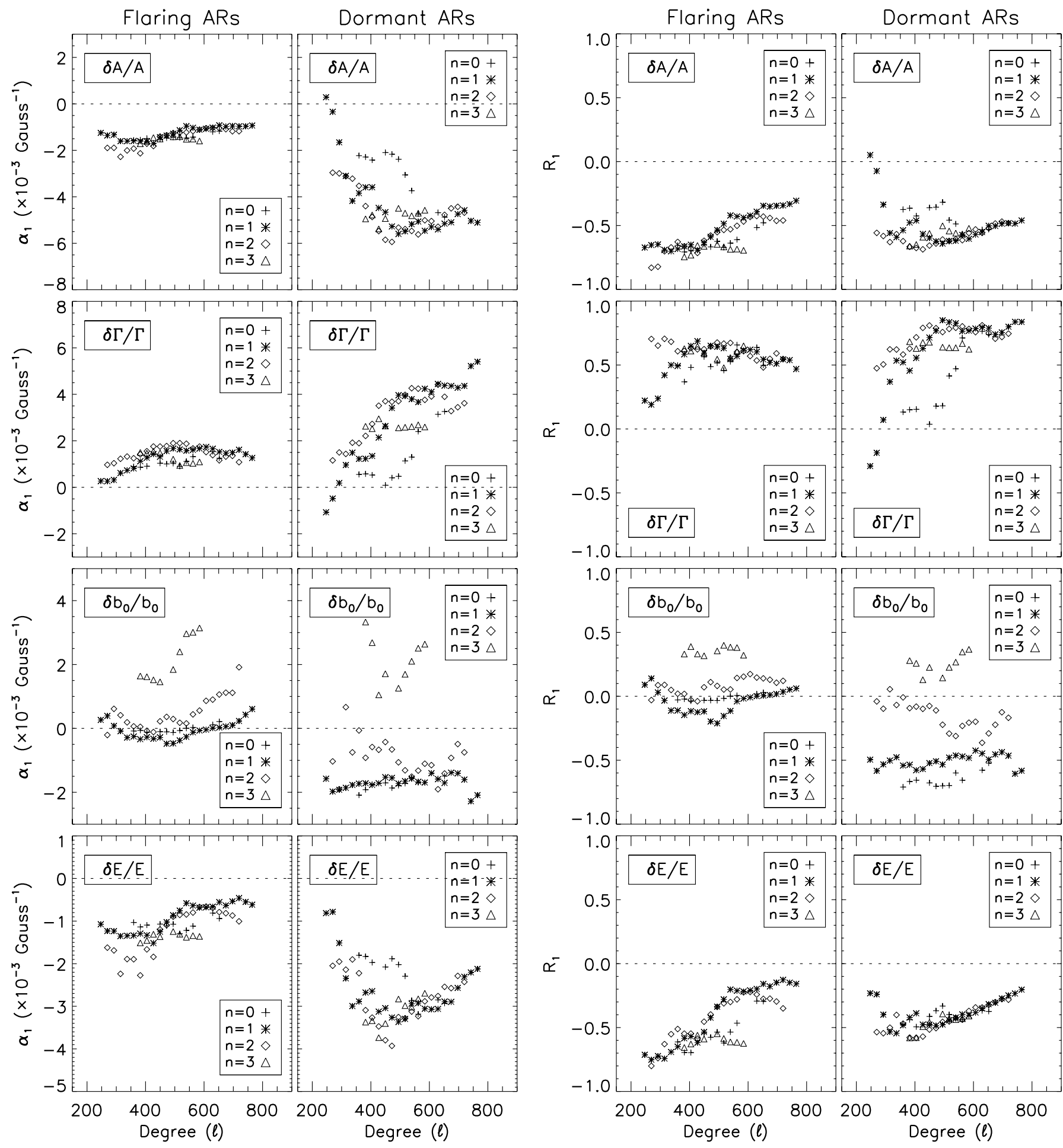

Fig. 10. Coefficients of linear regression in $\delta B$ (left two columns) and Pearson correlation (right two columns), for different mode parameters of flaring and dormant ARs as a function of harmonic degree.

restricted to the $\ell<200$, where the variation is weak even in our results.

The coefficients of linear regression $\left(\alpha_{1}\right)$ between the fractional difference in background power and $\delta B$ are shown in Figs. 10 and 11 (third row from the top) as a function of $\ell$ and $v$, respectively. The coefficient $\alpha_{1}$ is negative (positive) for most flaring (dormant) ARs with $n<2$. For p-modes of flaring ARs, it first decreases with frequency and becomes lowest at different frequencies in the five-minute band for different radial orders, and then increases. The negative value of the coefficients $\alpha_{1}$

furthermore shows the opposite relation between background power and MAI, $B$. The positive value of $\alpha_{1}$ for flaring ARs shows that power enhancement may be caused by flare induced excitation. However, the correlation coefficients for most of the modes are very low for flaring ARs.

\subsubsection{Mode energy}

If the total power in the mode is the area under the peak in the power spectrum, it is not instantly clear whether the increase in 

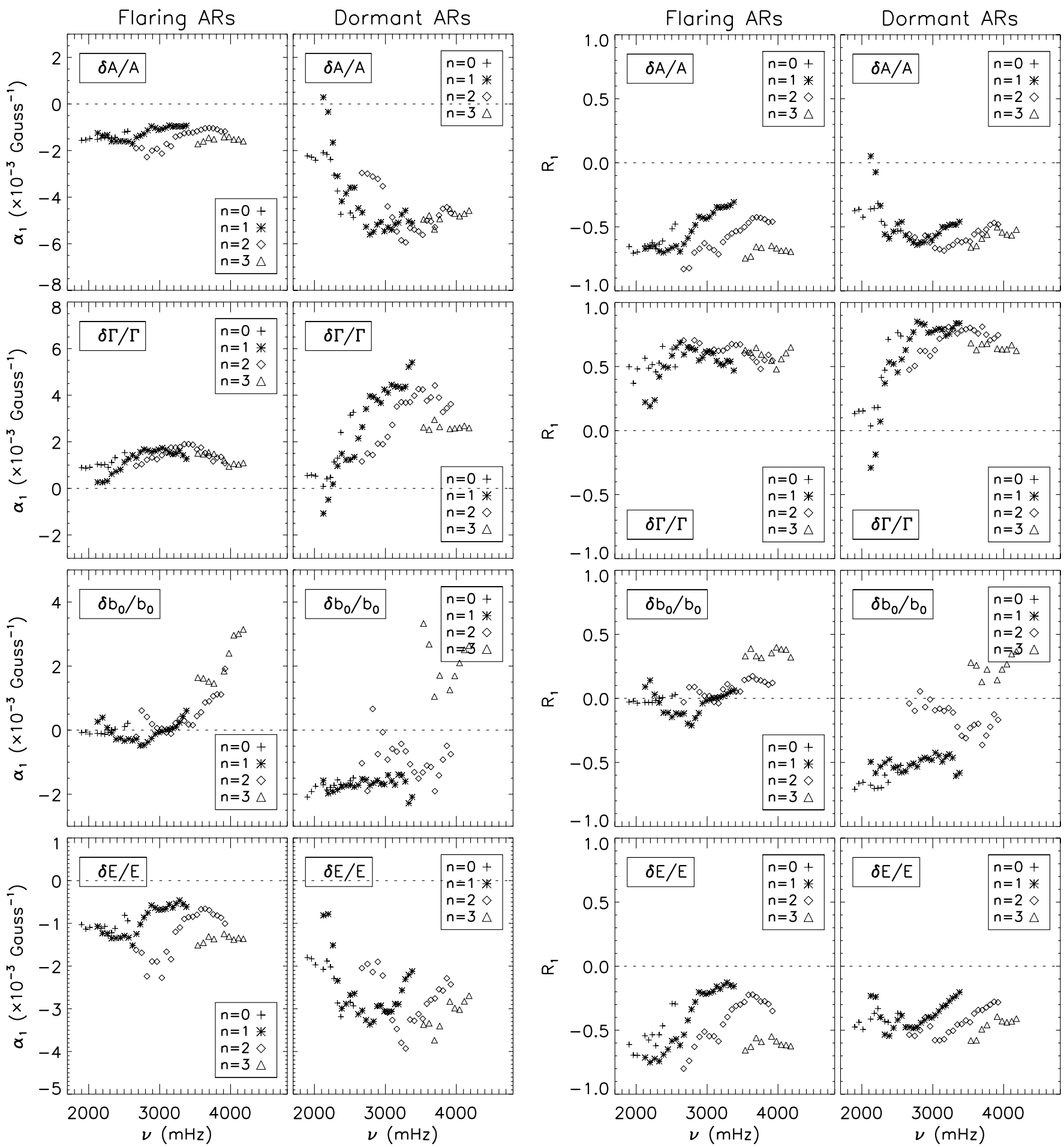

Fig. 11. Similar to Fig. 10, but as a function of frequency.

line widths in ARs compensates for the decrease in peak height. The area below a peak is a measure of excitation for the corresponding mode. To examine these changes, we plotted the fractional difference of the product of amplitude and width as a proxy for the area below the peak or mode energy (see Eq. (7)) in three frequency bands. This is shown in Fig. 9 (right two columns).

Figure 9 shows that the mode energy for both flaring and dormant ARs is lower than in the corresponding QRs and decreases with increasing $\delta B$. But the decrease rate in dormant ARs is faster than in flaring ARs, as is evident from the linear

regression slope (see Table 2). In the dormant ARs the energy difference decreases faster in the five-minute band than in the lower- and higher-frequency bands. The intercept $(a)$, and also the anti-correlation coefficient decrease from lower to higher frequencies. For the global modes, Komm et al. (2000a) reported a reduction of up to $60 \%$ in mode area. They found that the highest reduction of $36 \%$ in mode area occurs in the frequency range of $2.7-3.3 \mathrm{mHz}$.

The linear regression fit shows that the slopes of the flaring ARs are very small for the five-minute band. This implies that the mode energy slowly decreases with $B$ of flaring ARs. This is 


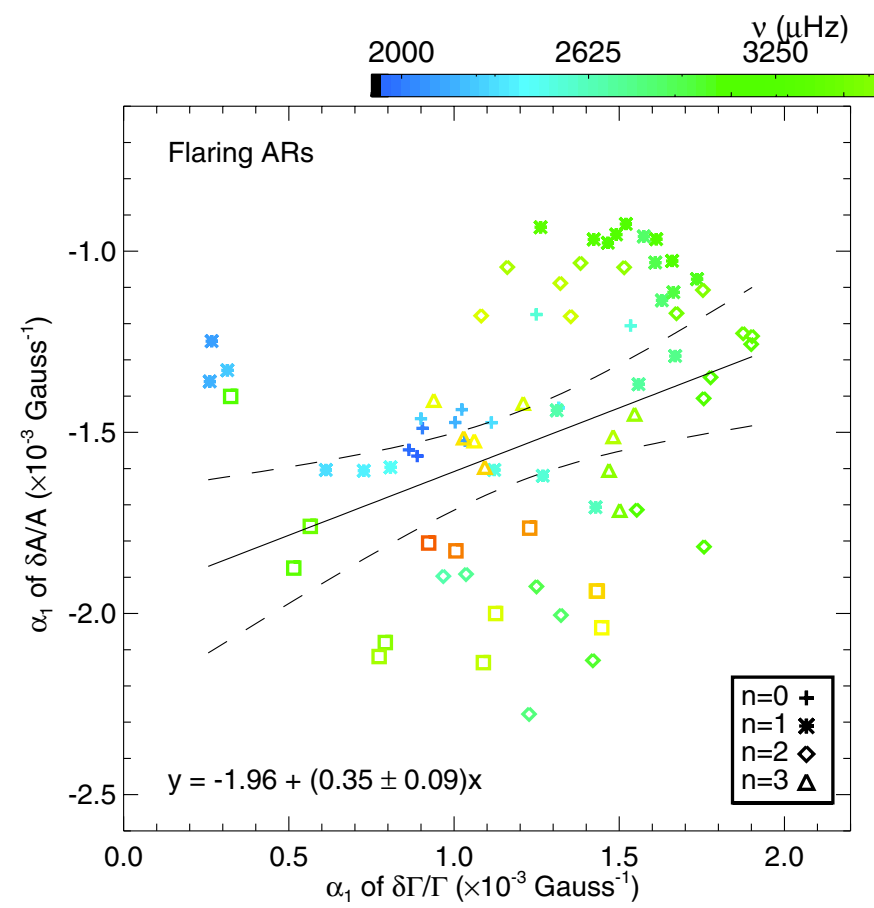
$3875 \quad 4500$

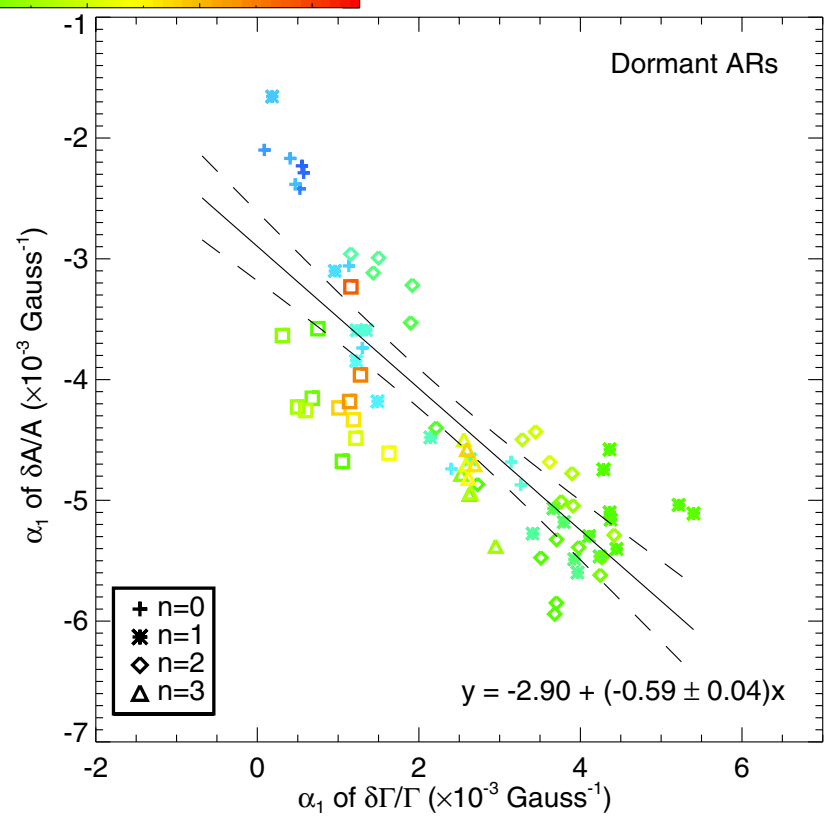

Fig. 12. Variations in the rate of fractional differences in mode amplitude and mode width of flaring (left) and dormant (right) ARs. Solid lines show the linear regression fit with the fitted coefficients as given in the respective equations. (This figure is available in color in electronic form.)

perplexing because several studies have reported strong p-mode power absorption by AR magnetic fields (Braun et al. 1987; Braun \& Duvall 1990; Rajaguru et al. 2001; Mathew 2008). Therefore, the mode energy is expected to decrease faster with increasing $B$ instead of the rate as, is inferred by the small slope for the energy difference for the flaring ARs in Fig. 9.

Figures 10 and 11 (bottom panel) show the coefficients of linear regression $\alpha_{1}$ between the fractional mode energy difference $\delta E / E$ and $\delta B$ as a function of harmonic degree and frequency, respectively. The energy variation rate (i.e., $\alpha_{1}$ ) for dormant ARs initially decreases with increasing degree $\ell$, and becomes lowest around $\ell \approx 500$ for all the radial orders then increases. We note that there is a contribution to the mode energy from the instrumental resolution, which is effectively around 5 arcsec for GONG due to seeing. Previous studies have shown a decrease in mode energy with harmonic degree at a fixed frequency (e.g. Rhodes et al. 1991). Woodard et al. (2001) have also studied the energy rate in the intermediate mode. They inferred that the time-averaged energy per mode, which is theoretically related to the modal surface velocity power, decreases steeply with $\ell$ at a fixed frequency over the entire observed $\ell$ range. Specifically, at $v=3.1 \mathrm{mHz}$, the energy per mode drops by a factor of $\sim 10$ between $\ell=150$ and $\ell=650$. For flaring ARs, the mode energy variation rate is significantly different from the dormant ARs. $\alpha_{1}$ for flaring ARs increases with increasing $\ell$ except for few modes. On average, $\alpha_{1}$ of flaring ARs is smaller in magnitude than in dormant ARs, indicating lower mode energy in flaring ARs than in dormant ARs.

The above-mentioned difficulty may be resolved as follows: Note that we computed the mode energy using data cubes corresponding to the ARs of our sample during their maximum flaring periods. Hence, the mode energy would include the net result of absorption due to the sunspots and amplification due to the flares. In ARs with a strong magnetic fields, that is, high magnetic index $(B)$, but lower flare-activity, that is, low flare index (FI), the effect due to mode absorption would dominate. On the other hand, when the flare energy is higher than the energy absorbed by sunspots, the amplification effect due to flares would dominate. We suggest that the small slope in p-mode energy (Fig. 9), which does not show a significant decrease with increasing $B$, could be attributed to the increasingly stronger flaring activity in the magnetically complex, flare-productive ARs. However, it is not clear whether the variations in $A$ or in $\Gamma$ are contributing more to the changes in mode energy. In the following, we attempt to analyse this problem in more detail.

To study the major contribution to the mode energy variation, we plot the coefficients of the fractional amplitude variation and width variation for flaring and dormant ARs (see Fig. 12). For dormant ARs, we find that the fractional increase in mode width is followed by the fractional decrease in mode amplitude, supporting previous reports (Rabello-Soares et al. 2008). However, the increase in width is slightly faster than the decrease in amplitude, as is evident from the negative slope of the linear regression. This shows that the fractional decrease in mode energy of dormant ARs is caused by the decrease in amplitude as well as in width, but the width increase contributes slightly more than the decrease in amplitude.

For the flaring ARs, the trend is quiet different from that of the dormant ARs. The increase in fractional difference in mode width is followed by the increase in fractional difference in mode amplitude and the resulting increase in fractional mode energy. This can be seen in Figs. 10 and 11 (bottom panel). The fractional increase in mode width is somewhat stronger than the fractional increase in mode amplitude. This shows that the net contribution to the variation in fractional mode energy is dominated by the mode width.

\subsection{Changes in mode parameters with flaring activity}

From the previous Sect. 4.1, we learned that the p-mode properties of flaring ARs are distinctly different from those of the dormant ARs. We have weaker mode suppression and lower 

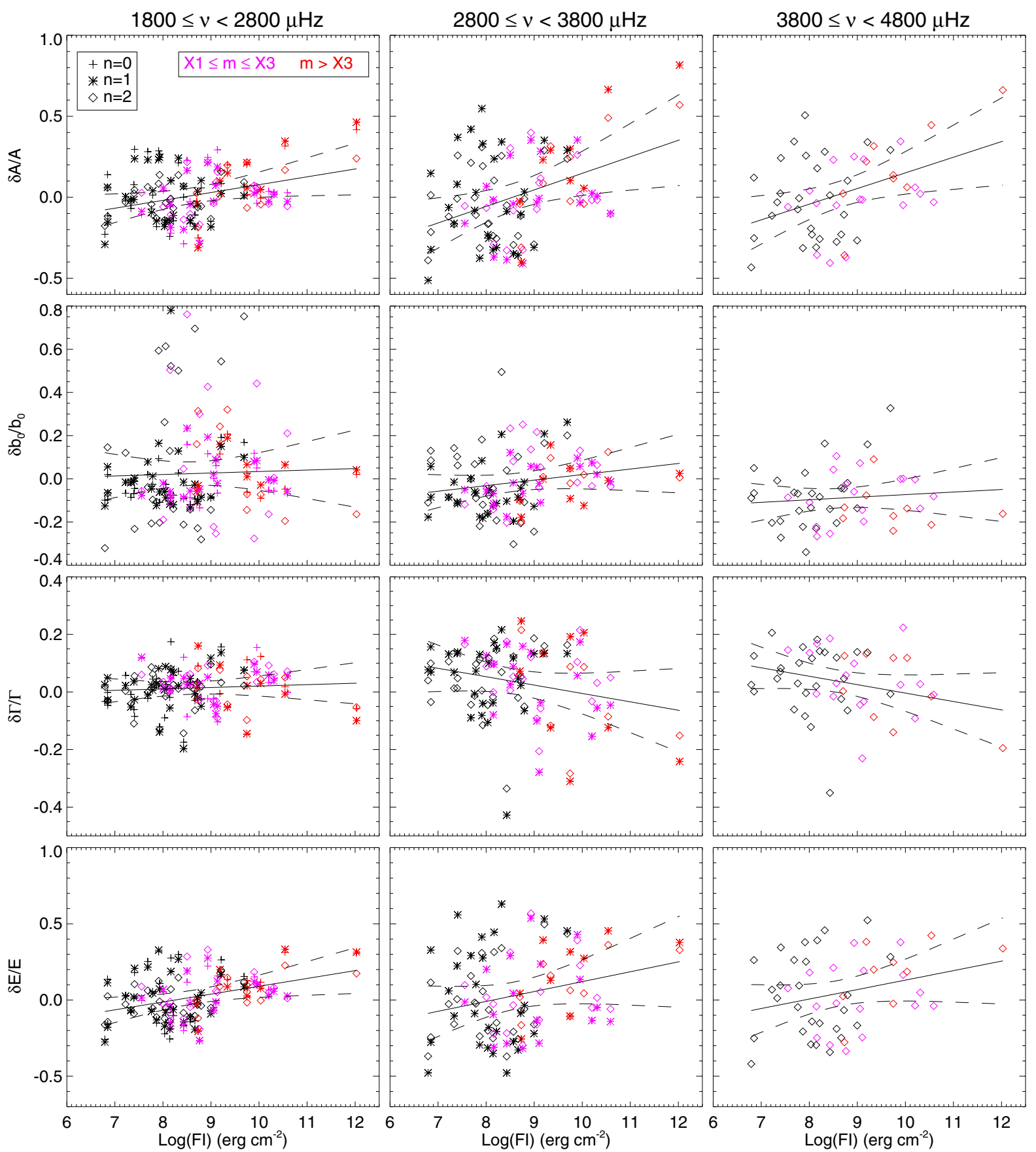

Fig. 13. Frequency averages of the fractional difference in mode amplitude, background power, mode width, and mode energy of flaring ARs, from top to bottom, as a function of flare-activity index. Mode parameters are corrected for foreshortening, duty cycle and magnetic-activity. Red and magenta symbols correspond to ARs flare(s) of magnitude $(m)>X 3$ and $X 1 \leq m \leq X 3$, respectively. The solid lines show the linear regression, and the dashed curves correspond to the $95 \%$ confidence level of the linear fit. (This figure is available in color in electronic form.)

width variation rates in flaring ARs than in dormant ARs. To study the flare-related changes in mode parameters, we corrected the mode parameters, of all the flaring ARs and corresponding QRs for foreshortening, duty cycle, and magnetic-activity. From Sect. 4.1 we found that the mode amplitude and width show a linear relation with $B$ in agreement with the reports by Rabello-Soares et al. (2008). We fitted the foreshortening- and duty-cycle-corrected mode parameters of all the ARs and QRs as a function of $B, \mathcal{P}_{n, \ell}^{\mathrm{c}}(B)=\xi_{0}+\xi B$. Then every mode parameter of flaring ARs and corresponding QRs were corrected for the MAI using the coefficients $\xi$ obtained from the fitting according to $\mathcal{P}_{n, \ell}^{\prime c}=\mathcal{P}_{n, \ell}^{\mathrm{c}}-\xi\left(B-B_{\min }\right)$, where $B_{\min }$ is the lowest value of $B$ in the data sample. We repeated this analysis for every common multiplet $(n, \ell)$ for mode parameters $A, b_{0}$, and $\Gamma$. Then we computed the fractional difference in mode parameters between flaring ARs and corresponding QRs (e.g., for mode energy see Eq. (7)).

Figure 13 shows the frequency average of the fractional mode difference of corrected mode-parameters as a function of the flare-activity index (FI). We computed the linear regression and correlation coefficients between the frequency averages of the fractional differences of mode parameters and flare indices, which are listed in Table 3. Figures 14 and 15 show the coefficients of linear regression and Pearson correlations 
R. A. Maurya et al.: Variations of p-mode amplitude, width, and energy in active regions
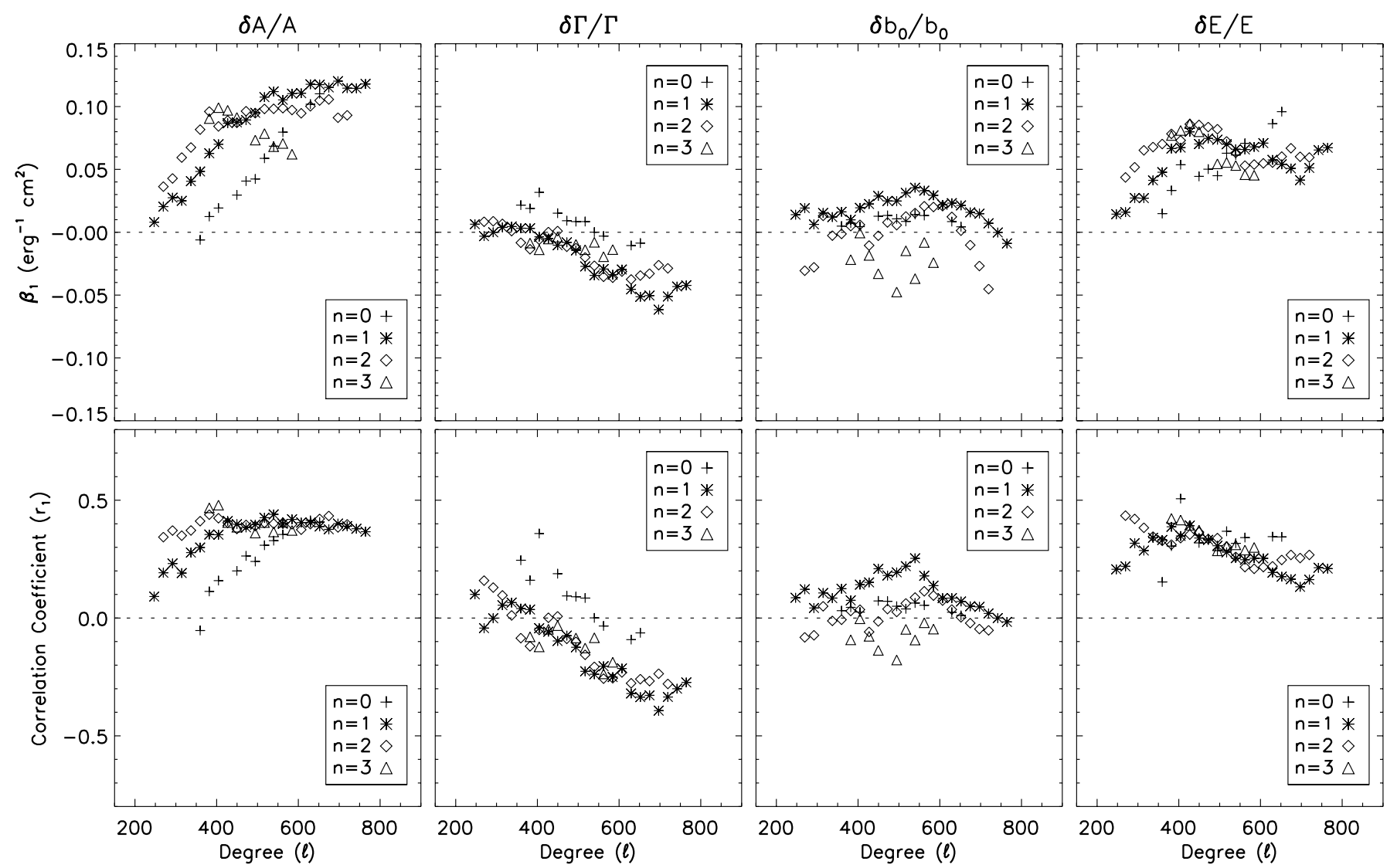

Fig. 14. Coefficients of linear regression in FI (top row) and Pearson correlation (bottom row) for different mode parameters of flaring ARs as a function of harmonic degree.

Table 3. Linear regression $(Y=\alpha+\beta \mathrm{FI})$ and Pearson correlation coefficients $(R)$ obtained from the frequency-averaged fractional mode difference $(Y)$ in three frequency bands and flare-activity index $(\mathrm{FI})$ of the flaring ARs.

\begin{tabular}{lcccc}
\hline \hline \multirow{2}{*}{$\begin{array}{l}\text { Mode } \\
\text { Parameters }\end{array}$} & $v$-range & $\alpha$ & $\beta \pm \sigma$ & $R$ \\
\cline { 2 - 5 } & $(\mu \mathrm{Hz})$ & \multicolumn{3}{c}{$\left(\times 10^{-2} \mathrm{erg}^{-1} \mathrm{~cm}^{2}\right)$} \\
\hline \multirow{3}{*}{$\delta A / A$} & $1800-2799$ & -0.41 & $4.84 \pm 1.79$ & 0.35 \\
& $2800-3799$ & -0.87 & $10.21 \pm 3.15$ & 0.41 \\
& $3800-4800$ & -0.82 & $9.66 \pm 3.04$ & 0.41 \\
\hline \multirow{3}{*}{$\delta b_{0} / b_{0}$} & $1800-2799$ & -0.04 & $0.69 \pm 2.02$ & 0.05 \\
& $2800-3799$ & -0.24 & $2.56 \pm 1.53$ & 0.23 \\
& $3800-4800$ & -0.19 & $1.19 \pm 1.67$ & 0.10 \\
\hline \multirow{3}{*}{$\delta \Gamma / \Gamma$} & $1800-2799$ & -0.03 & $0.50 \pm 0.81$ & 0.09 \\
& $2800-3799$ & 0.29 & $-2.91 \pm 1.63$ & -0.24 \\
& $3800-4800$ & 0.29 & $-2.93 \pm 1.46$ & -0.27 \\
\hline \multirow{3}{*}{$\delta E / E$} & $1800-2799$ & -0.42 & $5.15 \pm 1.69$ & 0.39 \\
& $2800-3799$ & -0.53 & $6.49 \pm 3.35$ & 0.26 \\
& $3800-4800$ & -0.49 & $6.22 \pm 3.16$ & 0.27 \\
\hline
\end{tabular}

Notes. The mode parameters are corrected for foreshortening, duty cycle, and magnetic-activity index.

between fractional difference in mode parameters and FI as a function of harmonic degree $(\ell)$ and frequency $(v)$, respectively. In the following, we analyse and discuss the various mode parameters.

\subsubsection{Mode amplitude}

Figure 13 (top panel) shows that the mode amplitude of flaring ARs increases with FI in all frequency bands. In the five-minute band the increase in $\delta A / A$ is stronger than the lower- and higherfrequency bands, as is evident from the linear regression slope for the five-minute band (see Table 3). Moreover, the correlation between $\delta A / A$ and FI is higher for the five-minute and higherfrequency bands than in the lower-frequency band. The positive steep slope shows flare-associated enhancement in mode amplitude. Our results of flare induced amplification in mode amplitude supports previous reports (Ambastha et al. 2003; Maurya et al. 2009).

However, several data points with $\log (\mathrm{FI}) \leq 9$ are seen below $\delta A / A=0$, showing a smaller amplitude in flaring ARs than in the corresponding QRs. Similar results have also been reported by Ambastha et al. (2003) for some flaring ARs. Figures 14 and 15 (left column) show the linear regression coefficient $\left(\beta_{1}\right)$ as a function of harmonic degree $(\ell)$ and frequency $(v)$, respectively. The amplitude variation rate increases with $\ell$ for all the radial orders. It increases with frequency and becomes highest in the five-minute band, then decreases. We also find a significant correlation for degree $\ell>300$ and $v>2500$.

\subsubsection{Mode width}

Figure 13 (third row from the top) shows the frequency average of the fractional difference $(\delta \Gamma / \Gamma)$ in mode width as a function of FI of the flaring ARs. $\delta \Gamma / \Gamma$ in the five-minute and higherfrequency bands decreases with increasing FI, while it shows 

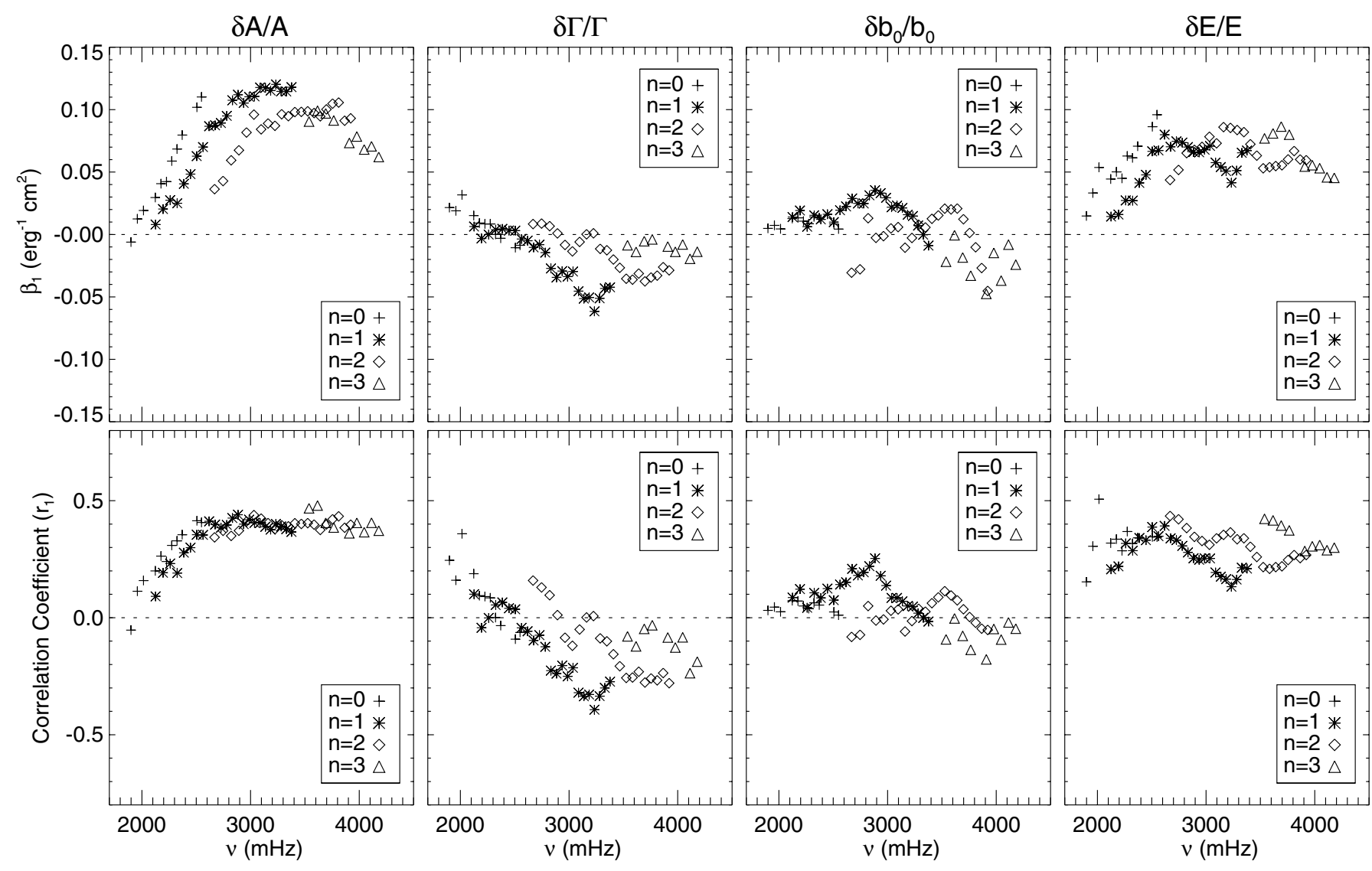

Fig. 15. Similar to Fig. 14, but as a function of frequency.

the opposite trend in the lower-frequency band. The relation between fractional difference in mode width and FI is also evident from the regression slope and the correlation coefficients given in Table 3. There is a strong correlation between relative width and FI for the five-minute and higher-frequency band, but the correlation is poor at the lower frequency band. Ambastha et al. (2003) have found a decrease in mode width during flares in some ARs, but no such signatures for some other flaring ARs in their data samples. Tripathy et al. (2008) have found that the CME-prone ARs having lower values of magnetic flux have a smaller line width than the QRs.

Figures 14 and 15 (second column from the left) show the linear regression coefficients $\beta_{1}$ for relative mode width as a function of harmonic degree and frequency, respectively. The coefficient $\beta_{1}$ is very low for harmonic degree $\ell<450$ and decreases with increasing $\ell(>450)$. The correlation coefficients $(r)$ also show a similar trend as the parameter $\beta_{1}$. The coefficient $\beta_{1}$ decreases with frequency for different radial orders (Fig. 15). But for the modes with radial order $n=1,2, \beta_{1}$ is smallest in the five-minute band at different frequencies, then it increases. The decrease in mode width with FI indicates an increase in the mode lifetimes.

\subsubsection{Background power}

Figure 13 (second row from the top) shows that the fractional difference $\left(\delta b_{0} / b_{0}\right)$ in background power increases with FI in all three frequency bands; the slope steepens with increasing frequency. The slope is steeper in the five-minute band than in lower- and higher-frequency bands, similar to the amplitude. The correlation coefficient is also higher in the five-minute frequency band than in the lower- and higher-frequency bands as is evident from Table 3. The positive slope at all frequency bands of the background power furthermore suggests the flare-induced amplification in mode power. But the correlation in the background power and FI are not significant. The background power variation with degree (Fig. 10) and frequency (Fig. 11) show similar relations.

\subsubsection{Mode energy}

The frequency-averaged fractional difference in mode energy $(\delta E / E)$ in all three frequency bands increases with increasing flare-activity index, see Fig. 13 (bottom row). The rate of increase in $\delta E / E$ for the five-minute frequency band is highest as is evident from the magnitude of the linear regression slope, which is smaller in the lower- and higher-frequency bands (see Table 3). This indicates that flare induced excitation is more significant in the five-minute bands than in the lower and higher frequencies. The significant positive correlation coefficients between mode energy and FI additionally support these results.

Figures 14 and 15 show that the mode energy variation rate with FI increases with increasing frequency and harmonic degree and peaks in the degree range 400-600 and in the fiveminute frequency band for all radial orders, then decreases at higher degree and frequencies, respectively. To ascertain the contribution of amplitude and width to the energy variation, we plot the width variation rate vs. amplitude variation rate in Fig. 16.

Figure 16 shows that mode amplitude (mode width) variation rate is positive (negative) for most of the modes, and an increase in mode width is followed by a rapid decrease in mode 


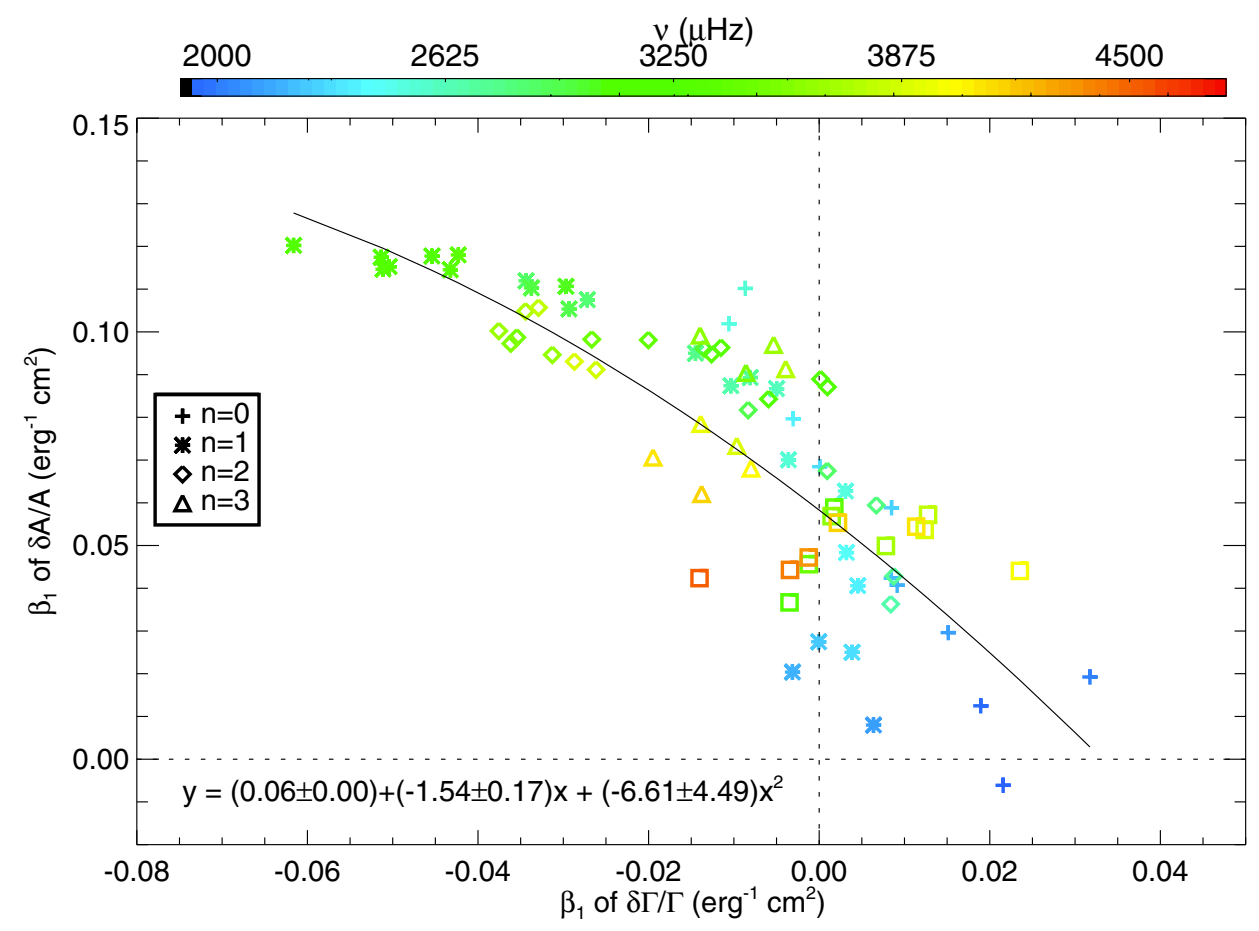

Fig. 16. Variations in the rate of fractional difference in mode amplitude and width of flaring ARs. The solid line shows the second-order polynomial fit; the fitted coefficients are given in the equation. (This figure is available in color in electronic form.) amplitude. This shows that the contribution of the mode amplitude to the variation of the mode energy is stronger than that of the mode width. In the five-minute frequency band, there is a high positive amplitude variation rate and a relatively low negative mode width rate. However, for a few modes in the lowerand higher-frequency bands the positive amplitude variation rate decreases very rapidly, and a positive mode width variation rate occurs.

\section{Summary and conclusions}

We studied the high-degree p-mode properties of a sample of several flaring and dormant ARs and associated QRs, observed during solar cycles 23 and 24 using the ring-diagram technique, assuming plane waves, and their association with magnetic and flare activities. The changes in p-mode parameters are the combined effects of duty cycles, foreshortening, magnetic and flare activities, and measurement uncertainties.

The p-mode amplitude $(A)$ and background power $\left(b_{0}\right)$ of ARs were found to be decreasing with their angular distances from the disc centre, while the width increases slowly. The effects of foreshortening on the mode amplitude and width are consistent with reports by Howe et al. (2004). The decrease in mode amplitude $A$ with distance arises because with increasing distance from the disc centre we measure only the cosine component of the vertical displacement. Moreover, foreshortening causes a decrease in spatial resolution of the Dopplergrams as we observe increasingly closer toward the limb. This reduces the spatial resolution determined on the Sun in the centre-to-limb direction, and hence leads to systematic observational errors.

The second-largest effects on p-mode parameters are caused by duty cycle. We found that the mode amplitude increases with increasing duty cycle, while the mode width and background power show the opposite trend. Similar results were reported previously for the global p-mode amplitude and width, for example by Komm et al. (2000a). These authors reported the strongest increase in mode width and reduction in amplitude with duty cycle when its values are lower. These changes in mode parameters may be caused by the increase in signal samples in data cubes. However, we found that for a few modes in the fiveminute and in higher-frequency bands, the mode amplitudes do not increase significantly with duty cycle. The effect of the duty cycle decreases with increasing harmonic degree $\ell$. To study the relation of mode parameters with magnetic and flare activities, we corrected the mode parameters of all the ARs and QRs for foreshortening.

We found that the mode amplitude in ARs is considerably smaller than in QRs. In the dormant ARs, the mode amplitude decreases with increasing $B$. There is a stronger reduction in the five-minute band than in the lower- and higher-frequency bands. The reduction in mode amplitude of ARs has been reported previously by several researchers (Braun et al. 1987; Braun \& Duvall 1990; Rajaguru et al. 2001; Mathew 2008). Goode \& Strous (1996) have reported the suppression of acoustic flux and p-mode power even in a weak magnetic field QR. However, a precise mechanism of the energy absorption is not yet established. The possible mechanisms for mode power reduction in ARs are (i) Absorption of p-modes within sunspots (Braun et al. 1988). It is assumed that the sunspot magnetic fields play a crucial role in transforming some part of the acoustic energy to pure magnetic energy, for instance, Alfvén waves type (Crouch \& Cally 2003, 2005; Cally et al. 2003), which may be transported to the upper atmosphere of the Sun (Marsh \& Walsh 2006, and references therein). (ii) The efficiency of the p-mode excitation by turbulent convection (Goldreich \& Keeley 1977; Goldreich \& Kumar 1988, 1990) might be reduced in a magnetic field owing to the nature of magnetoconvection (Hughes \& Proctor 1988). (iii) Wilson depression in a sunspot at the height of the formation of spectral lines, which is used to measure the Doppler shift, causes additional phase shifts to the velocity measurements, as compared to non-magnetic regions. (iv) Modification of the surface values of the p-mode eigenfunctions by the magnetic fields of ARs (Hindman et al. 1997). (v) Resonant absorption of solar p-modes by sunspots (Hollweg 1988). (vi) Mode mixing in sunspots (D’Silva 1994). 
The energy in an incoming mode, at any horizontal wavenumber, is dispersed into a wide range of wave numbers. (vii) Excitation of tube waves through p-mode buffeting (Bogdan et al. 1996; Hindman \& Jain 2008). (viii) Inhomogeneity-enhanced thermal damping (Riutova \& Persson 1984). Jain et al. (1996) showed that the horizontal magnetic field can lower the upper turning point and change the skin depth for a simple plane-parallel adiabatically stratified polytrope. In addition to power suppression, they also found that magnetic field alters the phase of p-modes.

The inclination of field lines from the vertical can affect the amount of acoustic power absorption (Cally et al. 2003) by conversion of acoustic to slow magneto-acoustic waves. The observational confirmation of the field inclination-related variations in mode power have been reported previously from the timedistance (Zhao \& Kosovichev 2006) and acoustic-holography (Schunker et al. 2005, 2008) analyses. In the ring-diagram analysis, we found these effects averaged over larger area.

The mode width in ARs are generally smaller than in the corresponding QRs and increases with magnetic field. This may be caused by strong damping of $\mathrm{p}$-modes in the strong magneticfield areas. Moreover, the width increases with frequency and becomes largest in the five-minute bands then decreases. But the relation in flaring ARs is poorer than in dormant ARs. This may be caused by flare-induced changes in mode parameters. For stochastically excited modes, a broadening in mode width shows a reduced lifetime or increased damping of the modes in regions of high magnetic-activity indices.

A possible mechanism by which magnetic-activity can influence mode widths is excitation of oscillations in flux tubes, as suggested by Bogdan et al. (1996) and Hasan (1997). These authors suggested that the flux tubes lead to a balance between energy input from p-modes and losses through radiative damping and leakage from flux-tube boundaries. The excitation of resonant oscillation in flux tubes in unstratified atmosphere was studied by Chitre \& Davila (1991) and Ryutova \& Priest (1993a,b). Resonant coupling with MHD waves (Pintér \& Goossens 1999) might also contribute to the damping of p-modes, as well as scattering of p-modes by the flux tube (Keppens et al. 1994; Bogdan $\&$ Zweibel 1987). Thus, when $B$ increases, p-modes are increasingly damped by the interactions with the increasing number of flux tubes. Gascoyne \& Jain (2009) have shown that the suppression of sound speed and pressure within the flux-tube region is not the only factor one needs to consider in the scattering of p-modes. There is a direct effect of the magnetic fields caused by the flaring of field lines on the phase shifts.

The combined effects of the mode amplification and width variations appear in mode energy variations. We found that the average mode energy in flaring ARs is lower at all frequencies than in dormant ARs and QRs. The decrease rate of the mode energy in dormant ARs is highest around $\ell=450$, while the highest decrease rate for flaring ARs shifted towards lower $\ell$. More interestingly, we found that the increase in width variation rate of dormant (flaring) ARs is followed by decrease (increase) in mode amplitude variation rate. The first one for the dormant ARs confirms previous reports by Rabello-Soares et al. (2008). The second one for the flaring ARs is attributed to the flare-associated changes in the oscillations characteristics of p-modes.

The decrease in mode energy implies a reduction in the amount of acoustic energy pumped into the modes by turbulent convection. This agrees with the assumption that the presence of strong magnetic fields suppresses motion in a turbulent medium, which is known to occur for several solar surface activities. For turbulent excitation and damping, the energy of a single oscillation mode is expected to depend mainly on mode frequency (at least for modes whose angular wavenumber is well below that of the excitation turbulence). The implication of energy depending only on frequency is that the surface velocity power of a single oscillation mode should increase with angular degree at fixed $v$. Theoretically, Jain et al. (2009) have shown that mode absorption increases rapidly with frequencyreaching a maximum or saturation value near $4 \mathrm{mHz}$, and decreases at higher frequencies $v>4 \mathrm{mHz}$. They investigated the p-mode absorption by fibril magnetic field. They suggested that the p-modes excite tube waves through mechanical buffeting on the magnetic fibrils in the form of longitudinal sausage waves and transverse kink waves. The tube waves propagate up and down the magnetic fibrils and out of the p-mode cavity, thereby removing energy from the incident acoustic waves.

The background power of the dormant ARs were found to be weaker than in corresponding QRs for radial order $n \leq 2$. But for the flaring ARs, the background power variations seem to be a function of harmonic degree and frequency $(v)$. It decreases with increasing $v$ and becomes lowest at a specific frequency in the five-minute frequency band then increases. These changes were attributed to the flare-associated charges in the oscillation modes. The background power of flaring and dormant ARs were found to decrease with MAI, which reinforces the idea that strong magnetic fields could hinder convection (Biermann 1941; Chandrasekhar 1961), which is the source of solar noise.

Magnetic-field-induced activities in the surface and higher layers may play a role in the excitation of oscillation modes in the ARs. To study the effects of flares on p-mode parameters, we employed mode corrections for foreshortening, duty cycle, and magnetic-activity. We found that the mode parameters show a significant correlation with flare-activity index. The pmode amplitude increases with flare-activity index and shows stronger amplification in the five-minute band. The increase in background power with flare-activity index furthermore supports the flare-associated excitation in p-modes (Wolff 1972). More interestingly, our statistical study showed an association of p-mode energy with flares, supporting the expected mode excitation by flares. The mode width is found to decrease with flare-activity index, indicating an increase in the lifetime of modes or mode damping. The combined effects of mode amplitude and width appeared in the mode energy. The mode energy increases with increasing flare-activity index and more strongly so in the fiveminute band. Our study showed that the increase in the mode energy is mostly contributed by the increase in mode amplitude and not by the increase in width or damping.

This suggests that the energetic solar flares may indeed produce effects in the solar interior below ARs, although the major process of flare-energy release occurs in the external solar atmosphere. However, the exact mechanism of the energy transfer towards the solar photosphere and flare induced excitation in mode power is not yet known. The detailed mechanism of the energy transfer from the corona to the photosphere is not well understood. Wolff (1972) was probably the first to propose the mechanism of momentum transfer toward the photosphere from the flare-energy release site to describe the possible impulse for the p-mode excitation. Recently, Hudson et al. (2012) proposed a similar mechanism of momentum transfer to explain flare-induced excitation of the seismic waves. They listed four basic mechanisms proposed previously: (i) a hydrodynamic shock-wave originating in the chromosphere (Kostiuk \& Pikelner 1975; Kosovichev \& Zharkova 1998); (ii) Lorentz force from the magnetic transients (Anwar et al. 1993; Kosovichev \& Zharkova 2001; Sudol \& Harvey 2005; Hudson et al. 2008); 
(iii) photospheric back-warming (Machado et al. 1989; Martínez-Oliveros et al. 2008); and (iv) "McClymont magnetic jerk" (Hudson et al. 2008). Work done by Lorentz forces in the back-reaction could supply enough energy to explain observations of flare-driven seismic waves (Hudson et al. 2008). The requirement for momentum conservation can in principle help to distinguish among these plausible mechanisms (Hudson et al. 2012). Hudson et al. (2008) introduced the idea of the coupling of flare energy into a seismic wave, namely the "McClymont magnetic jerk", produced during the impulsive phase of acoustically active flares.

Maurya \& Ambastha (2009) and Maurya et al. (2012) have reported large X-class white-light flares in seismically active ARs. Pedram \& Matthews (2012) have also studied the hard $\mathrm{X}$-ray characteristics of seismically active and quiet white-light flares. They found that the acoustically active flares are associated with a larger and more impulsive deposition of electron energy. However, they argued that this does not always correspond to a higher white-light contrast. Unfortunately, observations of white-light flares are rare. We suggest that the X-ray flux alone does not seem to be an adequate measure of how much energy a flare deposits in the photosphere. This is mainly because of the extent of soft X-ray emission produced at higher coronal heights. A lower X-class but larger $\mathrm{H} \alpha$-class or a white-light flare may be more important for affecting the solar acoustic oscillation modes.

We found that the flare index (FI), as calculated in this study, is a poor indicator for the helioseismic effects of a flare on the photosphere and on the oscillation characteristics because it is primarily based on the X-ray flux alone. Moreover, a higher FI value may result from several relatively longer-duration flares of smaller magnitude integrated over the time internal of the ring data cube. These small-magnitude flares that contribute to the larger FI may not be able to individually affect the oscillation characteristics. On the other hand, it is likely that a short-duration, energetic, and impulsive flare of large magnitude is seismically more effective, but may give rise to relatively smaller FI.

The MAI (MAI or $B$ ) used in this analysis was obtained from the line-of-sight components of the full magnetic field strength. The mode power absorption also depends upon the inclination of field lines from the vertical, as stated above. This may give rise to some systematic errors in the analysis of the relationship of mode parameters and magnetic-activity. Different magneticfield configurations in ARs may give different systematic errors. It is difficult to account for the order of the systematic errors, but continuous observations of vector magnetic fields can be used to analyse the errors.

This study supports previous results by Ambastha et al. (2003) and Maurya et al. (2009) that large flares are able to significantly amplify the high-degree p-modes, over and above the mode absorption by strong magnetic fields of ARs. The associated p-mode parameters are also affected by the flareinduced changes, thereby affecting the subsurface properties that are derived using the computed p-modes. Therefore, we suggest that adequate care should be taken in describing the subsurface properties of ARs especially while using the photospheric p-modes. We plan to analyse in depth the frequency shift, subphotospheric flow, sound speed, etc. in these active regions in our future studies.

Acknowledgements. This work used data obtained by the GONG program operated by AURA, Inc. and managed by the National Solar Observatory under a cooperative agreement with the National Science Foundation, USA The ringdiagram analysis performed using the GONG pipeline. The integrated X-ray flux data were obtained from GOES, which is operated by the National Oceanic and Atmospheric Administration, USA The solar activity information provided by the solar monitors web pages helped us to select the flaring and dormant active regions. The authors would like to thank H.M. Antia and F. Hill for their useful discussion and suggestions. Thanks are also due to the anonymous referee for his comments which helped in improving the paper. R.A.M. and J.C. acknowledge support by the National Research Foundation of Korea (2011-0028102 and NRF-2012R1A2A1A03670387).

\section{References}

Ambastha, A., \& Antia, H. M. 2006, Sol. Phys., 238, 219

Ambastha, A., Basu, S., \& Antia, H. M. 2003, Sol. Phys., 218, 151

Anderson, E. R., Duvall, Jr., T. L., \& Jefferies, S. M. 1990, ApJ, 364, 699

Antia, H. M., \& Basu, S. 2007, Astron. Nachr., 328, 257

Anwar, B., Acton, L. W., Hudson, H. S., et al. 1993, Sol. Phys., 147, 287

Biermann, L. 1941, Vierteljahrsschr. Astron. Ges., 76, 194

Bogdan, T. J., \& Zweibel, E. G. 1987, ApJ, 312, 444

Bogdan, T. J., Brown, T. M., Lites, B. W., \& Thomas, J. H. 1993, ApJ, 406, 723

Bogdan, T. J., Hindman, B. W., Cally, P. S., \& Charbonneau, P. 1996, ApJ, 465, 406

Braun, D. C., \& Duvall, Jr., T. L. 1990, Sol. Phys., 129, 83

Braun, D. C., Duvall, Jr., T. L., \& Labonte, B. J. 1987, ApJ, 319, L27

Braun, D. C., Duvall, Jr., T. L., \& Labonte, B. J. 1988, ApJ, 335, 1015

Burtseva, O., Hill, F., Kholikov, S., \& Chou, D. 2009a, Sol. Phys., 258, 1

Burtseva, O., Tripathy, S. C., Hill, F., et al. 2009b, in Solar-Stellar Dynamos as Revealed by Helio- and Asteroseismology: GONG 2008/SOHO 21, eds. M. Dikpati, T. Arentoft, I. González Hernández, C. Lindsey, \& F. Hill, ASP Conf. Ser., 416, 293

Cally, P. S., Crouch, A. D., \& Braun, D. C. 2003, MNRAS, 346, 381

Chandrasekhar, S. 1961, Hydrodynamic and hydromagnetic stability (Oxford: Clarendon)

Chaplin, W. J., Elsworth, Y., Isaak, G. R., Miller, B. A., \& New, R. 2000, MNRAS, 313, 32

Chen, K., Chou, D., \& TON Team. 1996, ApJ, 465, 985

Chitre, S. M., \& Davila, J. M. 1991, ApJ, 371, 785

Corbard, T., Toner, C., Hill, F., et al. 2003, in GONG+ 2002. Local and Global Helioseismology: the Present and Future, ed. H. Sawaya-Lacoste (Noordwijk: ESA), ESA SP, 517, 255

Crouch, A. D., \& Cally, P. S. 2003, Sol. Phys., 214, 201

Crouch, A. D., \& Cally, P. S. 2005, Sol. Phys., 227, 1

Donea, A.-C., \& Lindsey, C. 2005, ApJ, 630, 1168

Donea, A., Braun, D. C., \& Lindsey, C. 1999, ApJ, 513, L143

D'Silva, S. 1994, ApJ, 435, 881

Gascoyne, A., \& Jain, R. 2009, A\&A, 501, 1131

Goldreich, P., \& Keeley, D. A. 1977, ApJ, 212, 243

Goldreich, P., \& Kumar, P. 1988, ApJ, 326, 462

Goldreich, P., \& Kumar, P. 1990, ApJ, 363, 694

Goldreich, P., \& Murray, N. 1994, ApJ, 424, 480

Goode, P. R., \& Strous, L. H. 1996, Bul. Astron. Soc. India, 24, 223

Gosain, S., Mathew, S. K., \& Venkatakrishnan, P. 2011, Sol. Phys., 268, 335

Haber, D., Jain, R., \& Zweibel, E. G. 1999, ApJ, 515, 832

Haber, D. A., Hindman, B. W., Toomre, J., et al. 2002, ApJ, 570, 855

Harvey, J., Abdel-Gawad, K., Ball, W., et al. 1988, in Seismology of the Sun and Sun-Like Stars, ed. E. J. Rolfe (Noordwijk: ESA), ESA SP, 286, 203

Hasan, S. S. 1997, ApJ, 480, 803

Hill, F. 1988, ApJ, 333, 996

Hill, F., \& Howe, R. 1998, in Structure and Dynamics of the Interior of the Sun and Sun-like Stars, ed. S. Korzennik (Noordwijk: ESA), ESA SP, 418, 225

Hill, F., Bolding, J., Toner, C., et al. 2003, in GONG+ 2002. Local and Global Helioseismology: the Present and Future, ed. H. Sawaya-Lacoste (Noordwijk: ESA), ESA SP, 517, 295

Hindman, B. W., \& Brown, T. M. 1998, ApJ, 504, 1029

Hindman, B. W., \& Jain, R. 2008, ApJ, 677, 769

Hindman, B. W., Jain, R., \& Zweibel, E. G. 1997, ApJ, 476, 392

Hollweg, J. V. 1988, ApJ, 335, 1005

Howe, R., Komm, R. W., Hill, F., Haber, D. A., \& Hindman, B. W. 2004, ApJ, 608,562

Hudson, H. S., Fisher, G. H., \& Welsch, B. T. 2008, in Subsurface and Atmospheric Influences on Solar Activity, eds. R. Howe, R. W. Komm, K. S. Balasubramaniam, \& G. J. D. Petrie, ASP Conf. Ser., 383, 221

Hudson, H. S., Fletcher, L., Fisher, G. H., Abbett, W. P., \& Russell, A. 2012, Sol. Phys., 277, 77

Hughes, D. W., \& Proctor, M. R. E. 1988, Ann. Rev. Fluid Mech., 20, 187

Jain, R., Hindman, B. W., \& Zweibel, E. G. 1996, ApJ, 464, 476

Jain, R., Hindman, B. W., Braun, D. C., \& Birch, A. C. 2009, ApJ, 695, 325 
A\&A 561, A123 (2014)

Karoff, C., \& Kjeldsen, H. 2008, ApJ, 678, L73

Keppens, R., Bogdan, T. J., \& Goossens, M. 1994, ApJ, 436, 372

Komm, R. W., Howe, R., \& Hill, F. 2000a, ApJ, 531, 1094

Komm, R. W., Howe, R., \& Hill, F. 2000b, ApJ, 543, 472

Kosovichev, A. G. 2006, Sol. Phys., 238, 1

Kosovichev, A. G. 2011, ApJ, 734, L15

Kosovichev, A. G., \& Zharkova, V. V. 1998, Nature, 393, 317

Kosovichev, A. G., \& Zharkova, V. V. 2001, ApJ, 550, L105

Kostiuk, N. D., \& Pikelner, S. B. 1975, Sov. Astron., 18, 590

Kumar, B., Mathur, S., García, R. A., \& Venkatakrishnan, P. 2010, ApJ, 711, L12

Kumar, B., Venkatakrishnan, P., Mathur, S., Tiwari, S. K., \& García, R. A. 2011, ApJ, 743, 29

Leibacher, J. W., \& Stein, R. F. 1971, Astrophys. Lett., 7, 191

Leighton, R. B., Noyes, R. W., \& Simon, G. W. 1962, ApJ, 135, 474

Machado, M. E., Emslie, A. G., \& Avrett, E. H. 1989, Sol. Phys., 124, 303

Marsh, M. S., \& Walsh, R. W. 2006, ApJ, 643, 540

Martínez-Oliveros, J. C., Moradi, H., \& Donea, A.-C. 2008, Sol. Phys., 251, 613

Mathew, S. K. 2008, Sol. Phys., 251, 515

Maurya, R. A. 2010, Ph.D. Thesis, Mohan Lal Sukhadiya Univ., Udaipur, India

Maurya, R. A., \& Ambastha, A. 2009, Sol. Phys., 258, 31

Maurya, R. A., \& Ambastha, A. 2011, Astron. Soc. India Conf. Ser., 2, 189

Maurya, R. A., Ambastha, A., \& Tripathy, S. C. 2009, ApJ, 706, L235

Maurya, R. A., Vemareddy, P., \& Ambastha, A. 2012, ApJ, 747, 134
Pedram, E., \& Matthews, S. A. 2012, Sol. Phys, 277, 317

Pintér, B., \& Goossens, M. 1999, A\&A, 347, 321

Rabello-Soares, M. C., Bogart, R. S., \& Basu, S. 2008, J. Phys. Conf. Ser., 118, 012084

Rajaguru, S. P., Basu, S., \& Antia, H. M. 2001, ApJ, 563, 410

Rhodes, E. J., Cacciani, A., \& Korzennik, S. G. 1991, Adv. Space Res., 11, 17

Richardson, M., Hill, F., \& Stassun, K. G. 2012, Sol. Phys., 281, 21

Riutova, M., \& Persson, M. 1984, Phys. Scr., 29, 353

Ryutova, M. P., \& Priest, E. R. 1993a, ApJ, 419, 349

Ryutova, M. P., \& Priest, E. R. 1993b, ApJ, 419, 371

Scherrer, P. H., Bogart, R. S., Bush, R. I., et al. 1995, Sol. Phys., 162, 129

Schunker, H., Braun, D. C., Cally, P. S., \& Lindsey, C. 2005, ApJ, 621, L149

Schunker, H., Braun, D. C., Lindsey, C., \& Cally, P. S. 2008, Sol. Phys., 251, 341

Sudol, J. J., \& Harvey, J. W. 2005, ApJ, 635, 647

Tripathy, S. C., Wet, S., Jain, K., Clark, R., \& Hill, F. 2008, J. Astrophys. Astron., 29,207

Tripathy, S. C., Jain, K., Hill, F., \& Leibacher, J. W. 2010, ApJ, 711, L84

Ulrich, R. K. 1970, ApJ, 162, 993

Wolff, C. L. 1972, ApJ, 176, 833

Woodard, M. F., Korzennik, S. G., Rabello-Soares, M. C., et al. 2001, ApJ, 548, L103

Zhang, H. 1997, ApJ, 479, 1012

Zhao, J., \& Kosovichev, A. G. 2006, ApJ, 643, 1317 